\title{
Endtimes of Human Rights? Neue Forschungen zur Geschichte der Menschenrechte
}

\author{
Benjamin Möckel $\mathbb{D}$
}

Eingegangen: 10. September 2019 / Angenommen: 29. Juni 2020 / Online publiziert: 16. September 2020 (C) Der/die Autor(en) 2020

Zusammenfassung Die Menschenrechte sind in den letzten Jahren unter Druck geraten. Einige Autoren haben zuletzt sogar von einem „Ende“ der Menschenrechte als Leitlinie der internationalen Politik gesprochen. Der Artikel analysiert, wie neuere Forschungen der Menschenrechtsgeschichte auf diese Herausforderung reagieren. Er argumentiert, dass der lange dominierende Fokus auf Fragen der Periodisierung einem neuen Bewusstsein für die zeitliche, räumliche und thematische Pluralität und Heterogenität der Menschenrechte gewichen sei und beleuchtet vor diesem Hintergrund vier zentrale Forschungsfelder: erstens das Verhältnis von Menschenrechten und Ökonomie, zweitens die Geschichte humanitärer Interventionen und der hiermit einhergehenden Verbindung von Menschenrechten und militärischer Gewalt, drittens die Rolle der Medien in der Geschichte der Menschenrechte und viertens die neuen Herausforderungen einer Globalgeschichte der Menschenrechte.

Schlüsselwörter Menschenrechte · Globalgeschichte · Gerechtigkeit ·

Völkerrecht · Humanitäre Intervention · NGOs

\section{Endtimes of Human Rights? New Research in Human Rights History}

\begin{abstract}
Human Rights have come under pressure in recent years. Some scholars have even argued that we are witnessing the "endtimes of human rights". This article analyses how recent scholarship in human rights historiography has responded to this challenge. It argues that the focus on questions of periodization has been transcended into a new awareness for the multiplicity of times, places, and topics of human rights history. The article highlights four emerging research fields: first, the relationship of human rights and economics, second, the history of humanitarian interventions,
\end{abstract}

B. Möckel $(\bowtie)$

Historisches Institut, Universität zu Köln, Köln, Deutschland

E-Mail: bmoeckel@uni-koeln.de 
third, the role of media in human rights history, and fourth, the new challenges of a global history of human rights.

Keywords Human Rights · Global History · Justice · International law · Humanitarian intervention $\cdot$ NGOs

\section{Einleitung: ,Endtimes of Human Rights“،}

Glaubt man neueren Veröffentlichungen, so befinden wir uns seit einiger Zeit in den „Endtimes of Human Rights“. Stephen Hopgoods gleichnamiges Buch, das bei seiner Veröffentlichung im Jahr 2013 intensive Debatten auslöste, spiegelt in seinem Titel eine verbreitete Wahrnehmung: Menschenrechte, so der Tenor vieler aktueller Stimmen, haben ihren Status als leitendes Normsystem der internationalen Politik verloren. Nachdem Menschenrechtsaktivist_innen lange Zeit im Kielwasser einer wohlwollenden politischen Öffentlichkeit agiert hätten, würden sie nun eher gegen den politischen Zeitgeist ankämpfen. Menschenrechte, so die verbreitete Wahrnehmung, sind in die Defensive geraten. ${ }^{1}$

Seit der Veröffentlichung von Hopgoods Buch gibt es auf den ersten Blick kaum Anzeichen für eine Wiederbelebung. Die Brexitabstimmung im Sommer 2016 und die Wahl Donald Trumps wenige Monate später können als Zeichen einer Rückkehr nationaler Deutungsmuster und eines Bedeutungsverlustes multilateraler Ansätze in der internationalen Politik interpretiert werden. Kerninstitutionen des internationalen Menschenrechtssystems wie der Internationale Strafgerichtshof sehen sich mit Vorwürfen einer selektiven Strafverfolgung konfrontiert und sind durch Austritte oder Austrittsdrohungen einzelner Staaten unter Druck geraten. Die Europäische Union - 2012 mit dem Friedensnobelpreis ausgezeichnet - hat im Kontext ihrer Flüchtlingspolitik und unter dem Eindruck erstarkender nationalistischer Kräfte zentrale Verpflichtungen der Schutzverantwortung und des Asyls ausgehöhlt. Und gleichzeitig scheint es den etablierten Menschenrechtsorganisationen immer schwerer zu fallen, das Interesse der Medien auf menschenrechtspolitische Fragen zu lenken. Amnesty International beispielsweise erfuhr zuletzt nicht durch Kampagnen zu globalen Menschenrechtsverletzungen Aufmerksamkeit, sondern durch die kritische Berichterstattung über die eigenen Arbeitsbedingungen. ${ }^{2}$

Bei genauerer Betrachtung liegen die Kritiklinien jedoch tiefer und sind nicht erst aus den Verschiebungen in der internationalen Politik der vergangenen Jahre hervorgegangen. Vor allem drei Aspekte haben hier eine zentrale Rolle gespielt. Erstens hat sich in der Folge von 9/11 und den Kriegen in Afghanistan und im Irak die Kritik an einer Militarisierung der Menschenrechte und ihrer Instrumentalisierung für machtpolitische Zwecke verstärkt. Besonders die amerikanische Außenpolitik der Bush-

\footnotetext{
1 Hopgood, Stephen: The Endtimes of Human Rights, Cornell UP, Ithaca, NY 2013. Siehe z. B. auch Posner, Eric A.: The Twilight of Human Rights Law, Oxford UP, Oxford u. a. 2014.

2 Siehe z. B. McVeigh, Karen: Amnesty International Has Toxic Working Culture, Report Finds. Bullying, Public Humiliation and Discrimination Threaten Rights Group's Credibility, in: The Guardian, 6. Februar 2019.
} 
Ära wurde hierbei zu einem Menetekel der Menschenrechtsbewegung. Einerseits setzten sich die USA im Kontext des war on terror über zentrale Menschenrechtsverpflichtungen wie das Folterverbot und die Garantie eines fairen Gerichtsverfahrens hinweg. Andererseits rechtfertigten sie das eigene Vorgehen auch mit dem Verweis auf den Schutz der Menschenrechte, was von europäischen Politikern wie Tony Blair dankbar aufgegriffen wurde.

Den zweiten Kontext bildet die Finanzkrise seit dem Jahr 2008, in deren Folge Fragen von sozialer und ökonomischer Ungleichheit mit neuer Dringlichkeit auf die Tagesordnung gesetzt worden sind. ${ }^{3}$ Die Relevanz der Menschenrechte ist hierbei nicht direkt infrage gestellt worden, doch sah sich die Menschenrechtsbewegung nun verstärkt dem Vorwurf ausgesetzt, sie habe Fragen globaler sozialer Gerechtigkeit und ökonomischer Umverteilung lange Zeit vernachlässigt und dem Aufstieg neoliberaler Politikmuster aus diesem Grund kaum etwas entgegenzusetzen gehabt. Diese Debatte über das Verhältnis von Menschenrechtsbewegung und Neoliberalismus gewinnt gerade erst an Kontur. Klar ist jedoch, dass Fragen von globaler Gerechtigkeit und sozioökonomischen Rechten in den vergangenen Jahren eine sehr viel größere Aufmerksamkeit erhalten haben.

Drittens ist schließlich die postkoloniale Kritik an den Menschenrechten zu nennen, die eine weit zurückreichende Geschichte hat, aber in den vergangenen zwei Jahrzehnten noch einmal an Bedeutung gewonnen hat. Dies gilt für die akademischen Debatten, in denen der Universalismus der Menschenrechte auf seine (post-)kolonialen Traditionslinien, Deutungszuschreibungen und Machtasymmetrien untersucht worden ist. ${ }^{4}$ Es gilt - mit oft ganz anderen Intentionen - aber auch für die politische Sphäre, wo Vorwürfe des Neokolonialismus und Eurozentrismus von Politikern genutzt werden, um die Verbindlichkeit universeller Normen in kulturrelativistischer Perspektive zu hinterfragen. Diese Argumente sind zuerst prominent in den Debatten über sogenannte ,Asian values' in den 1990er Jahren formuliert worden und werden bis in die Gegenwart in unterschiedlichen Konstellationen aufgegriffen. ${ }^{5}$

Die Ausgangsfrage des vorliegenden Beitrags lautet, wie sich die aktuellen Forschungen der Menschenrechtsgeschichte in diesen Kontext einer kritischen Bestandsaufnahme der Menschenrechte einfügen. Denn „Endtimes of Human Rights“ heißt offensichtlich nicht notwendigerweise „Endtimes of Human Rights historiography“. Eher im Gegenteil: Der Strom neuer Veröffentlichungen reißt nicht ab, während das Thema zugleich alle Stadien einer erfolgreichen Etablierung als eigenständiges Forschungsfeld durchlaufen hat. Dennoch ist es eine offene Frage, wie sich die Menschenrechtsgeschichte im Kontext der skizzierten Veränderungen positionieren wird.

\footnotetext{
${ }^{3}$ Siehe als Ausgangspunkt dieser Debatten vor allem Piketty, Thomas: Das Kapital im 21. Jahrhundert, Beck, München 2014 (frz. 2013).

${ }^{4}$ Siehe vor allem die Arbeiten von Mutua, Makau: Human Rights. A Political and Cultural Critique, Pennsylvania UP, Philadelphia, PA 2008; Spivak, Gayatri Chakravorty: Use and Abuse of Human Rights, in: Boundary 2. An International Journal of Literature and Culture 32 (2005), H. 1, S. 131-189; dies.: Righting Wrongs. Unrecht Richten, diaphanes, Zürich/Berlin 2008.

5 Jenco, Leigh: Revisiting Asian Values, in: Journal of the History of Ideas 74 (2013), H. 2, S. 237-258.
} 
Zwei Gründe sprechen dafür, dass die Geschichtswissenschaft sogar noch eine größere Bedeutung erhalten könnte. Einerseits hat sich die Menschenrechtsgeschichte von Beginn an in Abgrenzung zu den triumphalistischen Narrativen früher Darstellungen und Diskussionen entwickelt. Teleologische Erzählungen eines unaufhaltsamen Aufstiegs der Menschenrechte, die in den 1990er Jahren unverkennbar auch die Dimension eines politischen Legitimationsdiskurses besaßen, sind einer kritischeren Sichtweise gewichen, in der Rückschläge, Aporien und Ambivalenzen eine größere Bedeutung erhielten. Diese Perspektiven scheinen heute aktueller denn je. Zweitens legt die Diskussion eines ,Endes` der Menschenrechte fast unweigerlich auch die Frage nach deren Beginn nahe und verschiebt den Diskurs von der Ebene des vermeintlich Überzeitlichem zum historisch Spezifischen. Nicht zufällig trägt eine für Ende des Jahres angekündigte Gesamtdarstellung der Menschenrechtsgeschichte den bezeichnenden Untertitel: „Ein Rückblick“. 6

Die Assoziation ist klar: Menschenrechte seien demnach zu einem Phänomen geworden, dessen politische Bedeutung weitgehend abgeklungen ist und damit in den Aufgabenbereich der Geschichtswissenschaft übergehen könne. Dies wäre in der Tat ein grundlegender Umbruch für ein zeitgeschichtliches Forschungsfeld, das sich stark durch den Austausch und die Reibung an politischen Debatten und einer interdisziplinären Forschung in Politikwissenschaft, Rechtswissenschaft und Soziologie definiert hat. Bislang hat es jedoch nicht den Anschein, als würde sich dies grundlegend ändern. Vieles spricht eher dafür, dass die Geschichte der Menschenrechte weiterhin ein ,heißes“ Thema bleibt, dessen Erforschung sich im direkten Austausch mit aktuellen politischen und gesellschaftlichen Debatten vollzieht. Die Menschenrechtsgeschichte steht dabei vor einer doppelten Herausforderung: Einerseits wird sie auch weiterhin in einem interdisziplinären Forschungszusammenhang stehen und die Erkenntnisse anderer Fachrichtungen in die eigene Forschung integrieren müssen. Andererseits gilt es aber auch, diese Diskussionen und Forschungsfelder selbst zu historisieren und als Teil des Menschenrechtsdiskurses zu untersuchen. Auch die Rede von den „Endtimes of Human Rights“ wird dann in Zukunft womöglich als Diskursformel einer spezifischen Diskussionskonstellation in der Geschichte der Menschenrechte zu historisieren sein.

\section{Menschenrechtsgeschichte seit ,The Last Utopia“}

Die Geschichte der Menschenrechte gehört zu den produktivsten Forschungsfeldern der Geschichtswissenschaft. Nachdem Historiker_innen das Thema erst relativ spät entdeckt haben, ist in den vergangenen zwei Jahrzehnten eine kaum zu überblickende Menge an Veröffentlichungen erschienen, sodass eine umfassende Darstellung der existierenden Forschung mittlerweile kaum mehr möglich ist. Ziel des vorlie-

\footnotetext{
${ }^{6}$ Hoffmann, Stefan-Ludwig: Geschichte der Menschenrechte. Ein Rückblick, Suhrkamp, Berlin 2020.
} 
genden Beitrags ist es somit nicht, einen vollständigen Literaturbericht zu geben ${ }^{7}$, sondern anhand ausgewählter Schwerpunkte wichtige Tendenzen und Perspektiven der aktuellen Forschung herauszuarbeiten. Der folgende Abschnitt gibt daher nur in größter Verdichtung einige Hinweise zur Forschungsdiskussion der vergangenen anderthalb Jahrzehnte.

Die Geschichte der Menschenrechte nahm etwa um die Jahrtausendwende Fahrt auf. Nach ersten Monografien, die zunächst hauptsächlich die langen Linien einer vermeintlichen Kontinuität globaler Verrechtlichung skizzierten ${ }^{8}$, sowie bis heute einflussreichen Aufsätzen von Mark Mazower und Kenneth $\mathrm{Cmiel}^{9}$, erschienen 2007 mit Lynn Hunts „Inventing Human Rights“ sowie 2010 mit Samuel Moyns „The Last Utopia“" zwei Bücher, die die Forschungsdiskussionen der folgenden Jahre in zentraler Weise prägten. ${ }^{10}$ Hunt stellte in ihrem Buch die Zeit der Französischen Revolution und insgesamt das (europäische) 18. Jahrhundert in den Mittelpunkt ihrer Analyse und interpretierte die Genese der Menschenrechte vor allem als Resultat eines Wandels von Vorstellungen von Subjektivität, Moral und Empathie, die eine neue Aufgeschlossenheit gegenüber dem Leid anderer Menschen hervorgebracht habe. Hieraus seien - etwa in der Anti-Sklaverei-Bewegung und den Kampagnen zur Abschaffung der Folter - Prozesse der Verrechtlichung hervorgegangen, deren Einfluss sich bis in die Gegenwart weiterverfolgen ließe.

In Abgrenzung hierzu bildete für Moyn die Betonung von Diskontinuität den zentralen analytischen Zugang. Während Hunt auf mentalitätsgeschichtlicher Ebene die langen Traditionslinien und übergreifenden Bezugspunkte in den Mittelpunkt stellte, betonte Moyn in ideengeschichtlicher Perspektive die Inkommensurabilität unterschiedlicher Vorstellungen von individuellen Rechten und argumentierte, dass sowohl das frühneuzeitliche Naturrecht als auch die Bürgerrechte des Zeitalters der Französischen Revolution kaum Verbindungen zum modernen Menschenrechtsdenken aufwiesen. Noch provozierender war seine These, dass auch der postwar moment der 1940er Jahre und die 1948 verabschiedete Allgemeine Erklärung der Menschenrechte für die internationale Politik beinahe folgenlos blieben. Nach seiner Interpretation waren es erst die 1970er Jahre, in denen die Menschenrechte -

\footnotetext{
7 Vgl. hierfür u. a.: Moyn, Samuel: Substance, Scale, and Salience. The Recent Historiography of Human Rights, in: Annual Review of Law and Social Science 8 (2012), H. 1, S. 123-140; Pendas, Devin O.: Toward a New Politics. On the Recent Historiography of Human Rights, in: Contemporary European History 21 (2012), H. 1, S. 95-111; Brier, Robert: Beyond the Quest for a "Breakthrough". Reflections on the Recent Historiography on Human Rights, in: Jahrbuch Für Europäische Geschichte 16 (2015), S. 155-173; Heerten, Lasse: Menschenrechte und Neue Menschenrechtsgeschichte, in: Docupedia-Zeitgeschichte, 31. Januar 2017, URL: <https://docupedia.de/zg/Heerten_menschenrechte_v1_de_2017> [Zugriff: 07.07.2020]; Kandler, Philipp: Neue Trends in der „,neuen Menschenrechtsgeschichte“, in: Geschichte und Gesellschaft 45 (2019), H. 2, S. 297-319.

${ }^{8}$ So z. B. Lauren, Paul Gordon: The Evolution of International Human Rights. Visions Seen, Pennsylvania UP, Philadelphia, PA 1998. Mit Fokus auf das Ende des Zweiten Weltkriegs als Ursprungserzählung siehe Glendon, Mary Ann: A World Made New. Eleanor Roosevelt and the Universal Declaration of Human Rights, Random House, New York 2001.

9 Mazower, Mark: The Strange Triumph of Human Rights. 1933-1950, in: The Historical Journal 47 (2004), H. 2, S. 379-398; Cmiel, Kenneth: The Emergence of Human Rights Politics in the United States, in: The Journal of American History 86 (1999), H. 3, S. 1231-1250.

${ }^{10}$ Hunt, Lynn: Inventing Human Rights. A History, Norton, New York 2007; Moyn, Samuel: The Last Utopia. Human Rights in History, Harvard UP, Cambridge, MA/London 2010.
} 
als „letzte Utopie“ - einen größeren Einfluss in Politik und Gesellschaft erlangen konnten.

Der Kontrapunkt der beiden Bücher bildet bis heute einen ausgezeichneten Einstieg in die Diskussion über eine Historisierung der Menschenrechte - gerade auch in der akademischen Lehre. In einer Relektüre beider Bücher drängt sich der Eindruck auf, dass sich die damalige Forschungsdiskussion viel zu schnell auf die Frage der Periodisierung verengt hat. Stattdessen würde es sich lohnen, die theoretischen und methodologischen Unterschiede der beiden Ansätze noch einmal produktiv in Dialog zu setzen: Insbesondere die thematischen Zugänge, die Lynn Hunt als eine der frühen Vertreterinnen der Neuen Kulturgeschichte für das 18. Jahrhundert entwickelt hat, bilden für die Zeitgeschichte weiterhin innovative Anknüpfungspunkte, die erst langsam entdeckt zu werden scheinen: Fragen nach der Mediengeschichte der Menschenrechte, nach visuellen und semantischen Darstellungsweisen, nach Emotionen, materiellen Dimensionen und nicht zuletzt auch nach einer Geschlechtergeschichte der Menschenrechte, sind bei ihr schon angelegt und geraten erst jetzt stärker in den Fokus der zeithistorischen Forschung.

In den nächsten Jahren differenzierte sich die Forschung in schnellen Schritten aus. Den besten Überblick hierüber lässt sich über die zahlreichen Sammelbände gewinnen, die in den darauffolgenden Jahren in deutscher und englischer Sprache erschienen sind. ${ }^{11}$ Zugleich wurden erste Gesamtdarstellungen veröffentlicht, wobei Jan Eckels „Die Ambivalenz des Guten“ zweifellos als das aktuell umfassendste Standardwerk des gesamten Forschungsfeldes gelten kann. ${ }^{12}$ Mehrere Reader und Quellensammlungen ergänzen diese Darstellungen und darüber hinaus sind vor allem in Deutschland in letzter Zeit äußerst hilfreiche digitale Text- und Quellensammlungen entstanden. ${ }^{13}$

${ }^{11}$ Siehe als Auswahl der wichtigsten Veröffentlichungen: Hoffmann, Stefan-Ludwig (Hrsg.): Moralpolitik. Geschichte der Menschenrechte im 20. Jahrhundert (Geschichte der Gegenwart, Bd. 1), Wallstein, Göttingen 2010; Eckel, Jan/Moyn, Samuel (Hrsg.): Moral für die Welt. Menschenrechtspolitik in den 1970er Jahren (Schriftenreihe der FRIAS School of History, Bd. 5), Vandenhoeck \& Ruprecht, Göttingen 2012; Iriye, Akira/Goedde, Peter/Hitchcock, William (Hrsg.): The Human Rights Revolution. An International History, Oxford UP, Oxford u. a. 2012; Frei, Norbert/Weinke, Annette (Hrsg.): Toward a New Moral World Order. Menschenrechtspolitik und Völkerrecht seit 1945 (Jena-Center. Geschichte des 20. Jahrhunderts. Vorträge und Kolloquien, Bd. 15), Wallstein, Göttingen 2013.

12 Eckel, Jan: Die Ambivalenz des Guten. Menschenrechte in der internationalen Politik seit den 1940ern, Vandenhoeck \& Ruprecht, Göttingen 2014. Das Buch ist zuletzt auch in überarbeiteter Form in englischer Sprache erschienen: ders.: The Ambivalence of Good. Human Rights in International Politics Since the 1940s, Oxford UP, Oxford u. a. 2019.

${ }^{13}$ Ishay, Micheline R. (Hrsg.): The Human Rights Reader. Major Political Essays, Speeches, and Documents from Ancient Times to the Present, Routledge, London/New York 2007; Fassbender, Bardo (Hrsg.): Quellen zur Geschichte der Menschenrechte. Von der Amerikanischen Revolution zu den Vereinten Nationen, Reclam, Stuttgart 2014; Fritzsche, Karl Peter: Menschenrechte. Eine Einführung mit Dokumenten, Schöningh, Paderborn u. a. 2016. Für Online-Tools, die auch für die akademische Lehre großen Nutzen bieten, siehe z. B.: Arbeitskreis Menschenrechte im 20. Jahrhundert (Hrsg.): Quellen zur Geschichte der Menschenrechte, URL: <https://www.geschichte-menschenrechte.de/> [Zugriff; 12.07.2019]; das Themenportal von Plath, Christoph: „Utopien im Wandel. Zur Geschichte der Menschenrechte im 20. Jahrhundert“, in: Zeitgeschichte-online, Dezember 2018, URL: <https://zeitgeschichte-online.de/themen/utopienim-wandel> [Zugriff: 12.07.2019]; Klose, Fabian u. a. (Hrsg): Online Atlas on the History of Humanitarianism and Human Rights, URL: <http://wiki.ieg-mainz.de/ghra/> [Zugriff: 12.07.2019]. 
Aus den seitdem erschienenen Monografien lassen sich einige Leitmerkmale herausarbeiten. In zeitlicher Perspektive haben sich in Anlehnung an Hunt und Moyn besonders drei Schwerpunkte herausgebildet: das 18. Jahrhundert, die 1940er Jahre und die 1970er Jahre, wobei Stefan-Ludwig Hoffmann zuletzt in einem intensiv diskutierten Beitrag das 19. Jahrhundert und die 1990er Jahre als weitere Schlüsselepochen ins Spiel gebracht hat. ${ }^{14}$ Die wichtigsten Akteure bildeten die UN, die Nationalstaaten, NGOs sowie einzelne Individuen und deren Einfluss auf die Menschenrechtspolitik. ${ }^{15}$ Thematisch standen unter anderem der Bereich von Gewalt, Genozid und Krieg ${ }^{16}$, die Bedeutung einzelner Menschenrechtskampagnen ${ }^{17}$, der Kontext von Kolonialismus und Dekolonisation ${ }^{18}$, sowie die Rolle des Kalten Krieges und dessen Endes im Zentrum. ${ }^{19}$

Konzeptionell ergeben sich hieraus unter anderem drei Leitlinien dieser ersten Welle der Menschenrechtsgeschichte. Erstens waren die Forschungen durch einen starken Fokus auf Fragen der Periodisierung gekennzeichnet, an denen sich die wichtigsten Kontroversen entzündeten. Zweitens waren viele Arbeiten durch das Streben nach einer möglichst klaren Definition der Menschenrechte und deren Abgrenzung von verwandten Konzepten wie des Naturrechts und der Bürgerrechte sowie benachbarten Forschungsfeldern wie der Geschichte des Humanitarismus und der Entwicklungspolitik geprägt. Und drittens folgten viele Arbeiten einer relativ starken Fokussierung auf politik- und diplomatiegeschichtliche sowie ideen- und begriffsgeschichtliche Zugänge. Im Folgenden werde ich zu zeigen versuchen, dass

14 Siehe den Artikel von Hoffmann und die beiden Repliken von Hunt und Moyn: Hoffmann, StefanLudwig: Human Rights and History, in: Past \& Present 232 (2016), H. 1, S. 279-310; Hunt, Lynn: The Long and the Short of the History of Human Rights, in: Past \& Present 233 (2016), H. 1, S. 323-331; Moyn, Samuel: The End of Human Rights History, in: Past \& Present 233 (2016), H. 1, S. 307-332.

15 Zur Menschenrechtspolitik innerhalb der UN siehe z. B. Mazower, Mark: Governing the World. The Rise and Fall of an Idea, Allen Lane, London 2012. Für nationalstaatliche Fallbeispiele siehe z. B. Keys, Barbara J.: Reclaiming American Virtue. The Human Rights Revolution of the 1970s, Harvard UP, Cambridge, MA/London 2014; Wildenthal, Lora: The Language of Human Rights in West Germany, Pennsylvania UP, Philadelphia, PA 2012.Für die Rolle von NGOs siehe u. a. Quataert, Jean H.: Advocating Dignity. Human Rights Mobilizations in Global Politics, Pennsylvania UP, Philadelphia, PA 2009; Hopgood, Stephen: Keepers of the Flame. Understanding Amnesty International, Cornell UP, Ithaca, NY 2006. Für die Rolle einzelner Individuen und biografische Zugänge zur Geschichte der Menschenrechte siehe Winter, Jay/Prost, Antoine: René Cassin and Human Rights. From the Great War to the Universal Declaration, Cambridge UP, Cambridge 2013.

16 Weinke, Annette: Gewalt, Geschichte, Gerechtigkeit. Transnationale Debatten über deutsche Staatsverbrechen im 20. Jahrhundert (Beiträge zur Geschichte des 20. Jahrhunderts, Bd. 19), Wallstein, Göttingen 2015; Power, Samantha: "A Problem From Hell". America and the Age of Genocide, Basic Books, New York 2013.

17 Drescher, Seymour: Abolition. A History of Slavery and Antislavery, Cambridge UP, Cambridge 2009; Fieldhouse, Roger: Anti-Apartheid. A History of the Movement in Britain. A Study in Pressure Group Politics, Merlin, London 2005.

18 Klose, Fabian: Menschenrechte im Schatten kolonialer Gewalt. Die Dekolonisierungskriege in Kenia und Algerien 1945-1962, Oldenbourg, München 2009; Burke, Roland: Decolonization and the Evolution of International Human Rights, Pennsylvania UP, Philadelphia, PA 2010.

19 Foot, Rosemary: The Cold War and Human Rights, in: Leffler, Melvyn P./Westad, Odd Arne (Hrsg.): The Cambridge History of the Cold War, Vol. 3: Endings, Cambridge UP, Cambridge 2010, S. 445-465; Snyder, Sarah B.: Human Rights Activism and the End of the Cold War. A Transnational History of the Helsinki Network, Human Rights in History, Cambridge UP, Cambridge 2011. 
diese drei Merkmale in den aktuellen Forschungen eher an Bedeutung verlieren und neue Ansätze vor allem dort zu finden sind, wo diese zeitlichen, thematischen und disziplinären Abgrenzungen aufgebrochen oder infrage gestellt werden.

Der Beitrag diskutiert insgesamt acht Neuerscheinungen, die einer solcher Neuverortung der Menschenrechtsgeschichte zugeordnet werden können. Im Mittelpunkt stehen vier Themenbereiche, die stellvertretend für zentrale Felder der aktuellen Forschung stehen: Das gilt erstens für das Feld der Ökonomie und die Frage nach den Verbindungen zwischen Menschenrechten und Konzepten globaler sozialer Gerechtigkeit, zweitens für das Feld von Krieg und Militär und die Debatten einer militärischen Durchsetzung von Menschenrechtsnormen im Kontext „humanitärer Interventionen“, drittens für die Rolle von Medien und Populärkultur, sowie viertens für neue globalgeschichtliche Ansätze mit Fokus auf den Einfluss nichtwestlicher Akteure auf die Herausbildung des Menschenrechtsregimes in der Zeit nach 1945.

\section{Menschenrechte, Ökonomie und Neoliberalismus}

Fragen von Ökonomie und globaler sozialer Gerechtigkeit haben in den letzten Jahren verstärkt Eingang in die Debatten der Menschenrechtsbewegung gefunden. Dies findet mittlerweile auch in der historischen Forschung Gehör, die langsam beginnt, den Verbindungen zwischen Menschenrechten und Ökonomie genauer nachzugehen. Bislang stand dabei vor allem die Wiederentdeckung der sozioökonomischen Rechte als der vermeintlich vergessenen Seite der Menschenrechtsgeschichte im Vordergrund. Diese Bemühungen haben zuletzt in ersten Aufsätzen, Tagungen und Forschungskooperationen ihren Niederschlag gefunden. ${ }^{20}$ Dies gilt ebenso für die Wiederentdeckung der socialist human rights, die nicht mehr allein als Leerfloskel in der Propaganda des Kalten Krieges weggeschoben, sondern auf ihre genuinen Ansätze, Ziele und Proklamationen untersucht werden. ${ }^{21}$

Im Kontext der generellen Renaissance von wirtschafts- und sozialgeschichtlichen Fragestellungen in der Geschichtswissenschaft lässt sich davon ausgehen, dass diese Ansätze in den kommenden Jahren weiter an Bedeutung gewinnen werden. Es ist insofern kein Zufall, dass zuletzt auch Samuel Moyn mit einem Buch hervorgetreten ist, das genau diesen Konnex von Ökonomie und Menschenrechten ins Zentrum stellt. ${ }^{22}$ Nachdem Moyn mit „The Last Utopia“ die frühe Forschungsdiskus-

\footnotetext{
20 Siehe z. B. die Veranstaltungen und Veröffentlichungen des ,Socioeconomic Rights in History Network“ an der University of Warwick (UK) sowie die zahlreichen Einzelbeiträge im „Dossier on Social Rights and Human Rights in the Twentieth Century“, in: Humanity. An International Journal of Human Rights, Humanitarianism, and Development 3 (2012), H. 3.

21 Siehe Betts, Paul: Socialism, Social Rights, and Human Rights: The Case of East Germany, in: Humanity. An International Journal of Human Rights, Humanitarianism, and Development 3 (2012), H. 3, S. 407-426; Smith, Mark B.: Social Rights in the Soviet Dictatorship. The Constitutional Right to Welfare from Stalin to Brezhnev, in: Humanity. An International Journal of Human Rights, Humanitarianism, and Development 3 (2012), H. 3, S. 385-406; sowie zuletzt: Richardson-Little, Ned: The Human Rights Dictatorship. Socialism, Global Solidarity and Revolution in East Germany, Cambridge UP, Cambridge 2019.

22 Moyn, Samuel: Not Enough. Human Rights in an Unequal World, Harvard UP, Cambridge, MA/London 2018.
} 
sion zur Geschichte der Menschenrechte entscheidend geprägt hat, kann man davon ausgehen, dass auch sein neues Buch „Not Enough“ einen immensen Einfluss auf künftige Forschungen haben wird.

Das gilt, obwohl oder gerade weil das Buch in zentralen Punkten von der in „Last Utopia“ formulierten Agenda abweicht. Während Moyn dort seine Definition der Menschenrechte bewusst eng gezogen hatte, um sie von früheren Konzepten universaler Rechte abzugrenzen, betont das neue Buch die fließenden Übergänge unterschiedlicher Formen der globalen Normsetzung. Und wohingegen Moyn im ersten Buch die Brüche und Diskontinuitäten seit der Französischen Revolution verdeutlicht hatte und die Geschichte der Menschenrechte letztlich auf die Jahrzehnte seit den 1970er Jahren beschränken wollte, so stehen nun eher die langen Kontinuitätslinien im Nachdenken über Formen der sozialen und ökonomischen (Un-)Gleichheit im Mittelpunkt.

Dies hat zur Folge, dass „Menschenrechte“ in weiten Teilen des Buches als ein ,anwesendes Abwesendes“ fungieren. Statt eine Geschichte der Menschenrechte ist das Buch eher eine Archäologie jener Vorstellungen globaler Gerechtigkeit, die nach Moyn in dem Menschenrechtsregime der 1970er Jahre keinen Platz fanden. Hieraus resultiert eine durchaus produktive Perspektiverweiterung zu den Thesen des ersten Buches: Während dort nur relativ abstrakt von konkurrierenden Utopien die Rede gewesen war, gegen die sich die Menschenrechte schließlich als moralischer Konsens durchgesetzt hätten, so steht nun genau jene Vielfalt und Heterogenität von Ansätzen, Konzepten und Projekten im Mittelpunkt, die seit dem 18. Jahrhundert das Denken über Formen sozialer Gerechtigkeit geprägt haben, bevor diese - das ist Moyns zentrale These - in den 1970er Jahren in dem Tandem aus human rights und basic needs als Leitlinien eines moralischen Minimalkonsenses aufgegangen seien.

Diese weit ausgreifende Perspektive ist die Stärke des Buches. Die Originalität von Moyns Beitrag besteht darin, dass er sich nicht auf eine bloße Wiederentdeckung der sozialen und ökonomischen Rechte beschränkt - hier bietet das Buch letztlich wenig Neues -, sondern eine sehr viel weitreichendere These entwickelt. Moyn unterscheidet zwischen equality und subsistence als zweier möglicher Zugänge für eine gerechte Verteilung von Ressourcen und argumentiert, das zentrale Versäumnis der Menschenrechtsbewegung habe darin bestanden, dass sie keinerlei Programm entwickelt habe, um Fragen sozialer Gleichheit in die eigene Agenda zu integrieren und sich daher als erschreckend kompatibel mit ökonomischen Entwicklungen einer radikalen Ausweitung sozialer Ungleichheit erwiesen habe.

Das Buch führt diese Argumentation in sieben inhaltlichen Kapiteln aus, die jeweils um spezifische Konstellationen der Abwägung zwischen Konzepten von Gleichheit und einer basalen Grundsicherung kreisen. Im ersten Kapitel stehen die Gerechtigkeitsdebatten im Kontext der Französischen Revolution im Mittelpunkt. Das zweite Kapitel betont die ökonomischen Dimensionen der Allgemeinen Erklärung der Menschenrechte von 1948, die bei Moyn hierdurch eine signifikante Aufwertung erhält. Das dritte Kapitel diskutiert die sozialpolitische Agenda Franklin D. Roosevelts während des Zweiten Weltkriegs und das vierte Kapitel wendet sich der globalen Dimension der Nachkriegsjahrzehnte zu und verdeutlicht an Beispielen wie Gunnar Myrdals Konzept der ,Welfare World“ und den Debatten über eine „Neue Internationale Ökonomische Ordnung“ (NIEO) die weitreichenden Vorschläge einer 
globalen Umverteilung, die in diesem Zeitraum kursierten. In Abgrenzung zu diesen zum Teil äußerst weit gefassten Konzepten globaler Gleichheit stellt Moyn in den folgenden beiden Kapiteln dar, wie seit den 1970er Jahren neue Ansätze Eingang in die internationale Politik fanden, die unter Konzepten wie „Basic Needs“ (Mahbub ul Haq) und „Basic Rights“ (Henry Shue) auf eine dem Individuum nicht verweigerbare Grundversorgung abzielten, frühere Vorstellungen einer umfassenden globalen Umverteilung jedoch weitgehend aufgaben.

Erst das letzte Kapitel nimmt die in der Einleitung formulierte Frage nach dem Verhältnis von Menschenrechten und Neoliberalismus wieder auf. Moyns Position bleibt hier zurückhaltend. Entgegen eher polemischer Proklamationen einer Komplizenschaft der Menschenrechtsbewegung mit dem Neoliberalismus, wie sie etwa von Naomi Klein formuliert worden sind ${ }^{23}$, ließen sich nach Moyn kaum direkte personelle und konzeptionelle Brücken zwischen beiden Bewegungen feststellen. Dennoch sei das gemeinsame zeitliche Auftreten nicht allein Zufall. Die Menschenrechte hätten sich - in ihrer Fokussierung auf das einzelne Individuum und der Betonung ziviler und politischer Rechte und Freiheiten - als ein Konzept erwiesen, das mit den ökonomischen Umverteilungen des Neoliberalismus erstaunlich kompatibel war und diesem auch nur wenig entgegenzusetzen hatte. Die Diskussion über das Verhältnis zwischen Menschenrechten und Neoliberalismus wird die Forschungsdebatten der kommenden Jahre noch weiter beschäftigen ${ }^{24}$, vermutlich in noch stärkerem Maße, wenn in absehbarer Zeit die 1990er Jahre als Untersuchungszeitraum in den Vordergrund treten. Ob sich dabei der Begriff des ,Neoliberalismus “ als analytischer Zugang bewährt, oder es eher darum gehen wird, die sehr viel ältere Frage nach dem Verhältnis von Menschenrechten und Kapitalismus neu in den Blick zu nehmen, wird sich dabei noch erweisen.

Weitere Veröffentlichungen, die Moyns eher essayistisch verfasstes Buch empirisch vertiefen, sind bislang noch Mangelware. Erste Anknüpfungspunkte finden sich jedoch bei Marco Duranti, dessen Dissertation sich mit der Europäischen Menschenrechtskonvention von 1950 (EMRK) auseinandersetzt, die er als Ausdruck einer „Conservative Human Rights Revolution“ interpretiert. ${ }^{25}$ Mit dem Blick auf die konservativen Ursprungspunkte des Menschenrechtsdiskurses greift Duranti eine Forschungsperspektive auf, die neben Samuel Moyn auch Lora Wildenthal bereits für die Bundesrepublik in den Blick genommen hat. ${ }^{26}$ Duranti formuliert jedoch eine neue Perspektive, indem er die Frage aufwirft, in welchem Verhältnis diese Initiativen $\mathrm{zu}$ den ökonomischen und sozialpolitischen Debatten in den europäischen Nachkriegsgesellschaften standen. Er spitzt dabei das Argument von Moyn

\footnotetext{
23 Klein, Naomi: The Shock Doctrine. The Rise of Disaster Capitalism, Palgrave Macmillan, Basingstoke/New York 2007, S. 146-159.

24 Siehe vor allem das für November 2019 angekündigte Buch von Whyte, Jessica: The Morals of the Market. Human Rights and the Rise of Neoliberalism, Verso, London 2019. Vgl. als ihre erste Veröffentlichung zum Thema dies.: Powerless Companions or Fellow Travellers? Human Rights and the Neoliberal Assault on Post-Colonial Economic Justice, in: Radical Philosophy 2 (2018), H. 2, S. 13-29.

25 Duranti, Marco: The Conservative Human Rights Revolution. European Identity, Transnational Politics, and the Origins of the European Convention, Oxford UP, Oxford u. a. 2017.

26 Moyn, Samuel: Christian Human Rights (Intellectual History of the Modern Age), Pennsylvania UP, Philadelphia, PA 2015; Wildenthal: Language (wie Anm. 15).
} 
zu: Nicht nur habe die Menschenrechtsbewegung das Feld von Ökonomie und sozialer Ungleichheit lange Zeit ignoriert. Vielmehr sei die Menschenrechtsrhetorik in der europäischen Nachkriegszeit genutzt worden, um die sozialpolitischen Projekte vieler europäischer Staaten durch transnationale Vereinbarungen auszubremsen.

Die Europäische Menschenrechtskonvention besitzt eine herausgehobene Stellung innerhalb der Menschenrechtsvereinbarungen der unmittelbaren Nachkriegszeit. Sie etablierte ein juristisch bindendes europäisches Menschenrechtssystem mit weitreichenden Implementierungsmechanismen wie der Möglichkeit von Individualpetitionen durch alle Staatsbürger der Mitgliedsländer. In der Forschung, so Duranti, werde sie jedoch häufig bloß als regionale Ausformulierung der Allgemeinen Erklärung der Menschenrechte von 1948 aufgefasst und in den Kontext eines Kampfes gegen Faschismus und Kommunismus eingeordnet. Dieser Interpretation widerspricht Duranti vehement. In Wirklichkeit habe es sich bei den beiden Menschenrechtserklärungen um ,counter projects“ (S. 321) gehandelt, die auf völlig unterschiedlichen Motivationen beruht hätten. Während die Erklärung der UN auf eine bis zur Französischen Revolution zurückreichende Tradition des Universalismus gründe, habe die europäische Konvention in erster Linie den Versuch einer Renaissance konservativer Konzepte von europäischer Zivilisation und christlicher Werte nach 1945 dargestellt.

Duranti kontextualisiert dies auf zwei zeitlichen Ebenen. Der erste Teil verdeutlicht die langen ideengeschichtlichen Traditionslinien, in denen es konservativen Akteuren vor allem darum gegangen sei, Menschenrechte als Signum einer,europäischen Zivilisation“ zu nutzen. Wie der Autor überzeugend darlegt, war die Europäische Menschenrechtskonvention hier in der Tat ein Gegenmodell zum Universalismus der Allgemeinen Menschenrechtserklärung: Bildete das Konzept der Menschenrechte bei der UN einen Modus, um Unterschiede zwischen Menschen und Kulturen in rechtlicher Hinsicht zu nivellieren, so fungierten sie im europäischen Kontext als Bekräftigung und moralische Legitimation der eigenen Abgrenzungen. Hierzu gehörte auch, dass die Konvention auf die europäischen Kolonialreiche explizit keine Anwendung fand.

Der zweite Teil rekonstruiert dann äußerst detailliert die konkreten Schritte, die konservative Akteure unternahmen, um die Konvention als Mittel einer Renaissance konservativer Politik - und im Fall von Winston Churchill kann man hinzufügen: konservativer Politiker - nach 1945 zu nutzen. Durantis Schwerpunkt liegt dabei auf Frankreich und Großbritannien, wobei er drei zentrale Kräfte ausmacht: Erstens die ,imperialists“ mit Churchill als Aushängeschild, die unter dem Schlagwort der ,europäischen Zivilisation“ eine europäische Sonderstellung und insbesondere das fortgesetzte Recht auf koloniale Herrschaftsausübung legitimieren wollten. Zweitens ein Kreis von „conservative Catholics“, die vor allem in Frankreich die Konvention nutzen wollten, um die Macht des säkularen Staates einzudämmen. Und drittens eine Gruppe von „,free-marketeers“, die besonders in Großbritannien ebenfalls auf den Menschenrechtsdiskurs zurückgriffen.

Der Kontext hierfür war der „social democratic moment“ (Tony Judt) der Nachkriegszeit und vor allem die weitreichenden sozialpolitischen Reformen der britischen Regierung unter Clement Attlee nach dessen Wahlsieg im Juli 1945. Britische Konservative verfolgten laut Duranti den Plan, mithilfe internationaler Vereinbarungen diesen sozialpolitischen Reformen rechtliche Grenzen zu setzen. So fanden 
sozioökonomische Rechte - im Gegensatz zur UN-Erklärung - in die europäische Konvention keine Aufnahme, während das „Recht auf Eigentum“ - welches in der Allgemeinen Erklärung der Menschenrechte nicht vorkam - explizit in einem Zusatzartikel in die Konvention aufgenommen wurde.

Auf der Basis von Durantis Darstellung bleibt es etwas in der Schwebe, als wie relevant diese ,neoliberale ' Interpretation der Menschenrechtspolitik der Nachkriegszeit einzuschätzen ist. Churchill als zentraler Repräsentant der Bewegung machte einige Andeutungen in diese Richtung, war in seinem Engagement aber kaum an den Detailfragen der zeitgenössischen Sozialpolitik interessiert. Stattdessen ist es mit David Maxwell Fyfe ein Politiker aus der zweiten Reihe, der von Duranti als wichtigster Repräsentant dieser Verbindung von Menschenrechten, Neoliberalismus und Totalitarismustheorie vorgestellt wird. Mit dem Wahlsieg Churchills im Jahr 1951 jedenfalls verlor die Begeisterung für transnationale Vereinbarungen schnell an Bedeutung. Nun waren die britischen Interessen wieder am wirkungsvollsten in 10 Downing Street aufgehoben.

In längerer Perspektive spricht zumindest vieles dagegen, dass die Europäische Menschenrechtskonvention zu einem Instrument neoliberaler Wirtschaftspolitik wurde. Im Gegenteil wird in aktuellen Veröffentlichungen durchaus intensiv darüber diskutiert, inwiefern die EMRK auch für sozialpolitische Fragestellungen genutzt werden kann. ${ }^{27}$ Darüber hinaus spart Duranti in seiner Darstellung weitgehend aus, dass es innerhalb des Europarats zur selben Zeit auch eine substanzielle Beschäftigung mit sozialen Rechten gab, die 1961 in die Europäische Sozialcharta mündete. Dies verdeutlicht, dass die Diskussion um die Menschenrechte auch innerhalb des Europarats vielschichtiger war, als es Duranti in seiner Fokussierung auf die konservativen Kräfte nahelegt.

Das ändert allerdings nichts daran, dass das Buch mehrere wichtige Herausforderungen für künftige Forschungen beinhaltet. In der aktuellen politischen Situation wird vermutlich am meisten Interesse finden, mit wie viel Verve sich britische Konservative in den frühen Nachkriegsjahren in ein Projekt der europäischen Zusammenarbeit stürzten. Zweitens eröffnen die dargestellten ökonomischen Kontexte eine zentrale Betrachtungsweise, die für die 1960er und 1970er Jahre fortgeführt werden müsste. Vor allen Dingen aber gilt es, Durantis an vielen Stellen angedeutete Perspektive weiterzuverfolgen, nach der Menschenrechte als ein Mittel angesehen werden müssen, mit deren Hilfe nicht nur Gleichheit hergestellt werden sollte, sondern in anderen Konstellationen auch politische, kulturelle und ökonomische Privilegien geschützt und legitimiert wurden.

\section{Menschenrechte und humanitäre Interventionen}

Ein zweites Feld, das die Debatten der vergangenen zwei Jahrzehnte stark geprägt hat, ist die Frage nach der Legitimität militärischer Mittel in der Menschenrechts-

\footnotetext{
27 Siehe z. B. das Kapitel „Socio-Economic Rights and the European Convention on Human Rights“, in: Londras, Fiona de/Dzehtsiarou, Kanstantsin: Great Debates on the European Convention on Human Rights (Palgrave Great Debates in Law), Palgrave Macmillan, Basingstoke/New York 2018.
} 
politik. Dem Minimalismus der Ziele, den Moyn als ein Charakteristikum der Menschenrechte herausgearbeitet hat, steht demnach vor allem seit dem Ende des Kalten Krieges ein Maximalismus der Mittel gegenüber, in dem Menschenrechtsverletzungen zu einem völkerrechtlich anerkannten Grund für ein militärisches Eingreifen und eine Verletzung der nationalen Souveränität geworden sind. Spätestens mit den Balkankriegen und der Verabschiedung der „Responsibility to Protect“-Vereinbarung der UN im Jahr 2005 hat dies auch Eingang in die internationale Politik gefunden. Seit einiger Zeit erscheinen nun auch erste Arbeiten, die diese Debatten in eine historische Perspektive einordnen.

Dabei lassen sich momentan zwei Forschungsschwerpunkte erkennen. Einerseits erfahren derzeit die 1990er Jahre als wichtige Epoche einer - nun auch militärisch unterfütterten - Menschenrechtspolitik größere Aufmerksamkeit. Unter dieser Perspektive entstehen aktuell erste Ansätze, die humanitären Interventionen dieses Zeitraums in einen zeithistorischen Kontext einzufügen. Zweitens sind in den vergangenen Jahren mehrere Arbeiten erschienen, die sich in einer längeren zeitlichen Perspektive mit den Ursprüngen humanitärer Interventionspraktiken auseinandersetzen und dabei insbesondere das 19. Jahrhundert als Ausgangspunkt in den Blick nehmen.

Der von Norbert Frei, Daniel Stahl und Annette Weinke herausgegebene Sammelband „Human Rights and Humanitarian Intervention“ konzentriert sich in erster Linie auf die Umbruchphase der 1990er Jahre, fügt diese jedoch zugleich in längere ideengeschichtliche Konstellationen im 20. Jahrhundert ein. ${ }^{28}$ In ihrer Einleitung formulieren die Herausgeber_innen vier Umstände, die für eine solche zeithistorische Kontextualisierung aufschlussreich seien: Erstens die Transformation der (Neuen) Linken in der Zeit nach den 1960er Jahren, zweitens die Weiterentwicklung des internationalen Völkerrechts, drittens die Genese der Menschenrechte als neuer Leitlinie der internationalen Politik sowie viertens ein Generationswechsel in Politik und Medien, den sie vor allem an führenden Politikern der Babyboomer-Generation festmachen. Auf diese Weise gelingt es dem Band, die Interventionen der 1990er Jahre in längere historische Kontexte einzuordnen. Zugleich verdeutlichen die empirischen Beiträge, dass die geschichtswissenschaftliche Beschäftigung mit dem Zeitraum noch relativ am Anfang steht. Eine quellengesättigte Darstellung der im Band immer wieder angeführten Fallbeispiele (Kosovo, Afghanistan, Somalia) findet man bislang kaum; hier wird eher das Feld für zukünftige Forschungen abgesteckt.

Dagegen ist es eine große Stärke des Buches, einen dezidiert interdisziplinären Ansatz zu verfolgen. Im Zentrum stehen vier Zugänge, die erstens auf die politischen Kontexte, zweitens auf die intellektuellen und akademischen Diskurse, drittens auf mediale Darstellungen sowie viertens auf die völkerrechtlichen Debatten und Entwicklungen fokussieren. Der Band vereint dabei auch methodisch sehr unterschiedliche Zugänge, von politik- und ideengeschichtlichen Ansätzen über kultur- und medienwissenschaftliche Beiträge, völkerrechtliche Texte bis zu praxisnahen Erfahrungsberichten von Journalist_innen. Besonders erhellend ist dabei, dass aus diesem

${ }^{28}$ Frei, Norbert/Stahl, Daniel/Weinke, Annette (Hrsg.): Human Rights and Humanitarian Intervention. Legitimizing the Use of Force Since the 1970s (Schriftenreihe Menschenrechte im 20. Jahrhundert, Bd. 2), Wallstein, Göttingen 2017. 
multidisziplinären Zugang eine sehr viel weiter gefasste Definition dessen entsteht, was eigentlich als ,Intervention“ zu verstehen sei. Das verleiht dem Band zwar einen etwas heterogenen Gesamteindruck, bildet aber zugleich eine äußerst produktive Irritation gegenüber eines bislang vor allem auf den militärischen Bereich fokussierten Verständnisses von ,Interventionen“. Während es in den Abschnitten zu Politik und Völkerrecht eher konventionell um das Feld militärischer Interventionen geht, folgen die Beiträge aus den Bereichen Wissenschaft und Medien einem sehr viel breiteren Konzept von ,Interventionen“, das zum Beispiel auch den Einfluss westlicher Wissensregime in postkolonialen Kontexten oder Medienkampagnen und deren Bildsprachen integriert.

Eine solche Erweiterung des Interventionsbegriffs könnte in der Tat ein sinnvoller Zugang sein, um die Spezifik der 1990er Jahre herauszuarbeiten, ohne für die Zeit nach 1989 von einer ,Stunde Null` humanitärer Interventionen auszugehen. Womöglich liegt ein entscheidender Punkt darin, dass ,Interventionen " - gerade in der NordSüd-Richtung - immer schon einen wichtigen Aspekt der internationalen Politik darstellten und in ihren Ursprüngen bis in die Zeit des Kolonialismus zurückverfolgt werden müssen. Die Spezifik der 1990er Jahre läge dann eher darin, dass sich mit dem Ende des Kalten Krieges ein Zeitfenster öffnete, in dem solche Interventionen auch als offene militärische Operationen durchgeführt und auf der internationalen Bühne mit Argumenten des Menschenrechtsschutzes legitimiert werden konnten.

Eine zweite Phase, in der dies in ähnlicher Weise geschah, war das 19. Jahrhundert. In der Forschung zu Ideen und Praxis der ,humanitären Intervention“ ist dieser Zeitraum daher verstärkt ins Zentrum der Aufmerksamkeit gerückt. Davide Rodognos 2011 erschienene Monografie über die humanitären Interventionen im Osmanischen Reich war hierfür ein erster Meilenstein. ${ }^{29}$ Im selben Jahr erschienen auch ein Sammelband und kurz darauf eine von Alexis Heraclides und Ada Dialla verfasste Gesamtdarstellung. ${ }^{30}$ Fabian Klose hat im Jahr 2015 ebenfalls einen Sammelband vorgelegt, der die Forschungsdebatten aufgreift und bis in die Gegenwart fortschreibt. ${ }^{31}$ Von ihm liegt nun auch eine Monografie zum Thema vor, die zugleich seine Habilitationsschrift darstellt. ${ }^{32}$

Den Ausgangspunkt für Kloses Arbeit bilden die aktuellen Debatten über Legitimität und Praxis humanitärer Interventionen. Er greift völkerrechtliche und politikwissenschaftliche Perspektiven auf, kritisiert aber, dass diese das Konzept meist nur in Bezug auf die Zeit seit den 1990er Jahren diskutieren würden. Im Gegensatz hierzu argumentiert er, dass die Genese des Konzeptes sehr viel weiter zurückreiche. In Wirklichkeit, so Klose, sei das 19. Jahrhundert als das ,Jahrhundert der humanitären Intervention“ anzusehen, in dem das Konzept ,an verschiedenen Schauplätzen

\footnotetext{
29 Rodogno, Davide: Against Massacre. Humanitarian Interventions in the Ottoman Empire 1815-1914, Princeton UP, Princeton, NJ/Oxford 2011.

30 Simms, Brendan/Trim, David J. B. (Hrsg.): Humanitarian Intervention. A History, Cambridge UP, Cambridge 2011; Heraclides, Alexis/Dialla, Ada: Humanitarian Intervention in the Long Nineteenth Century. Setting the Precedent, Manchester UP, Manchester/New York 2015.

31 Klose, Fabian (Hrsg.): The Emergence of Humanitarian Intervention. Ideas and Practice from the Nineteenth Century to the Present, Cambridge UP, Cambridge 2015.

32 Ders.: „In the Cause of Humanity“. Eine Geschichte der humanitären Intervention im langen 19. Jahrhundert, Vandenhoeck \& Ruprecht, Göttingen 2019.
} 
in Afrika, Asien, Europa und Amerika entstand, sich im kolonialen wie imperialen Kontext verfestigte und schließlich Eingang in zentrale Völkerrechtstexte fand“ (S. $15 \mathrm{f}$.).

Damit fügt sich Klose nahtlos in die angeführten Forschungen von Rodogno und anderen ein. Allerdings gibt er eine andere Antwort auf die Frage, wann und in welchem historischen Kontext der Ursprungspunkt der humanitären Intervention zu suchen ist. Galt bislang meist das europäische Eingreifen in den griechischen Befreiungskrieg als Ausgangspunkt, an den weitere Interventionen vor allem im Osmanischen Reich anschlossen, so verlagert Klose den Fokus zeitlich weiter in die Vergangenheit und auf einen anderen Schauplatz: „Nicht der Schutz christlicher Minderheiten“, so seine These, ,,sondern die internationalen Maßnahmen gegen [...] den transatlantischen Sklavenhandel werden zu Beginn des 19. Jahrhunderts als entscheidender Ausgangspunkt betrachtet [...]." Der besonders vom britischen Empire lancierte Kampf gegen den Sklavenhandel bildet für ihn daher den „Urtyp der humanitären Intervention“ (S. 29).

Diese Fokusverschiebung kommt auch in der Anlage des Buches zum Ausdruck, das in drei Abschnitte geteilt ist. Nachdem der erste Abschnitt auf breiter Literaturbasis die Genese neuer Vorstellungen von internationaler Kooperation und Intervention entwickelt, stellt der zweite Teil äußerst detailliert und quellennah Idee und Praxis des Abolitionismus und der Bekämpfung des transatlantischen Sklavenhandels dar, bevor der dritte Absatz weitere Beispiele humanitärer Interventionen der europäischen Großmächte sowie der USA behandelt und äußerst überzeugend die Verschränkung zwischen humanitären Motiven und Formen des Kolonialismus und Imperialismus herausarbeitet.

Kern der Arbeit bilden somit die Abschnitte zum transatlantischen Sklavenhandel. In diesen liegt auch die größte Innovationskraft der Publikation. Während viele Ausführungen zur Genese des Internationalismus im 19. Jahrhundert und den Interventionen im Osmanischen Reich schon an anderen Stellen diskutiert wurden und man insbesondere die imperiale Geschichte der USA mit dem vermeintlichen Umschlagpunkt des Jahres 1898 in einer aktuellen Veröffentlichung noch prägnanter zugespitzt lesen kann $^{33}$, sind die Abschnitte zum Sklavenhandel gefüllt mit neuen Erkenntnissen, die ebenso detailliert analysiert, gut erzählt wie in die relevanten politischen, diplomatischen, medialen und völkerrechtlichen Diskussionen eingebettet sind. Vor allem gelingt es Klose an vielen Stellen, die theoretischen Debatten zumindest in Ansätzen mit der konkreten Praxis der Implementierung zu verbinden, mit denen besonders Großbritannien nach Verabschiedung des „Slavery Act“ von 1807 versuchte, über bilaterale Verträge und sogenannte "Courts of Mixed Commissions“ eine internationale Durchsetzung des Verbots des Sklavenhandels zu erreichen.

Selbstverständlich bedeutet die Fokusverschiebung auf die Anti-Sklaverei-Bewegung jedoch nicht nur eine Neuperiodisierung, sondern sie geht auch mit einer grundlegenden inhaltlichen Deutungsverschiebung einher. Während die Interventionen im Osmanischen Reich von Beginn an auf eine deutliche Grenzziehung zwischen den außereuropäischen Gesellschaften als Ort der Intervention und den christlichen

33 Immerwahr, Daniel: How to Hide an Empire. A History of the Greater United States, Farrar, Straus and Giroux, New York 2019. 
Minderheiten als zu schützende Rechtssubjekte verwiesen, so steht mit dem Kampf gegen den Sklavenhandel nun jenes Beispiel im Mittelpunkt, das - bei allen Ambivalenzen und realpolitischen Erwägungen - im 19. Jahrhundert am ehesten als Ausdruck eines humanitären Universalismus verstanden werden kann. Wie weit trägt folglich Kloses Argument, den britischen und später internationalen Kampf gegen den Sklavenhandel als Ursprung von Konzept und Praxis der humanitären Intervention $\mathrm{zu}$ interpretieren?

Auf den ersten Blick fallen eher die Unterschiede ins Auge. Klose selbst verweist auf Definitionen aus dem Feld der Politikwissenschaften, nach denen humanitäre Interventionen als äußere Einmischung in die inneren Angelegenheiten eines anderen Staates zu verstehen seien, die meist für einen begrenzten Zeitraum auf militärische und andere gewaltförmige Mittel zurückgreifen. Für die britischen Kampagnen gegen den Sklavenhandel trifft das nur eingeschränkt zu. Ein Eingriff in die nationale Souveränität lag kaum vor, weil die meisten britischen Kampagnen auf offener See stattfanden, sodass die Frage nationaler Souveränitätsrechte nur im Kontext der Kontroversen um ein Durchsuchungsrecht einzelner Schiffe aufkam. Auch die Gewaltdimension lässt sich nur in Ausnahmefällen wie der Belagerung der Stadt Algier im Jahr 1816 nachweisen - charakteristischerweise also wiederum in einem außereuropäischen Zusammenhang, in dem die Befreiung christlicher Sklaven in einer muslimischen Umgebung im Mittelpunkt stand. Und auch die begrenzte Dauer trifft im Fall der über mehrere Jahrzehnte aufrechterhaltenen britischen Kampagnen gegen den Sklavenhandel nur bedingt zu.

Aktuelle Definitionen von humanitären Interventionen sind für die Kampagnen gegen den Sklavenhandel demnach nur begrenzt anwendbar. Das ändert aber nichts daran, dass Kloses Plädoyer für eine Integration des Abolitionismus in die Diskussionen zu den humanitären Interventionen des 19. Jahrhunderts eine äußerst sinnvolle und überzeugende Perspektiverweiterung darstellt. Denn obwohl die Kampagnen heutigen Definitionen einer humanitären Intervention nur begrenzt entsprechen, kann Klose überzeugend zeigen, dass ihr Einfluss auf spätere Interventionspraktiken dennoch von großer Bedeutung war. Das gilt auf zwei Ebenen: Erstens weist Klose darauf hin, dass der Kampf gegen den Sklavenhandel als Katalysator für neue Konzepte der internationalen Kooperation und Intervention fungierte. Er beeinflusste die zeitgenössischen völkerrechtlichen Debatten über Strategien und Möglichkeiten des politischen Eingreifens, schuf konkrete Mechanismen der internationalen $\mathrm{Zu}-$ sammenarbeit und der Implementierung internationaler Normen, und prägte nicht zuletzt auch massiv die öffentliche Meinung zu Fragen des Rechts und der Pflicht zum Eingreifen in Fällen internationaler Rechtverletzungen.

Zweitens fungierten die Kampagnen gegen den Sklavenhandel auch als eine direkte Referenz, auf die sich in Debatten über die folgenden Interventionen in Griechenland und dem Osmanischen Reich bezogen wurde. Das galt vor allem für Großbritannien, das diesen Interventionen zunächst äußerst skeptisch gegenüberstand, sich aber bald sowohl innen- als auch außenpolitisch mit dem Argument konfrontiert sah, dass man es sich in Anbetracht des eigenen Engagements gegen den Sklavenhandel nicht erlauben konnte, nun bei anderen internationalen Verbrechen abseits zu stehen. Für die britische Politik entstanden hieraus bestimmte Pfadabhängigkeiten der moralischen Aufladung der eigenen Außenpolitik, wie man sie ähnlich auch 
im 20. Jahrhundert für die internationale Menschenrechtspolitik beobachten kann. Gerade diese Dynamik gehört zu den stärksten Aspekten des Buches. Klose gelingt es hier, die meist wenig produktive Dichotomie zwischen humanitären und realpolitischen Motiven zu überwinden. Stattdessen scheint bei ihm ein komplexeres Argument durch, welches auch für die Menschenrechtspolitik der vergangenen Jahrzehnte von Bedeutung ist: das Phänomen nämlich, dass humanitäre Motive und Argumente in Politik und Öffentlichkeit des 19. Jahrhunderts eine gesteigerte Bedeutung erhielten und auf diese Weise selbst realpolitischen Einfluss erlangten.

\section{Menschenrechte und Medien}

Der letztgenannte Aspekt deutet auf einen Zusammenhang hin, der in den aktuellen Forschungen zur Menschenrechtsgeschichte eine immer größere Bedeutung erhält: die Rolle medialer Diskurse und Öffentlichkeiten, die sowohl in der Menschenrechtspolitik als auch in der Forderung nach humanitären Interventionen eine entscheidende Rolle spielten. Die Einbettung der Menschenrechte in mediale Konjunkturen und Darstellungsweisen bildet daher ein zentrales Feld der Menschenrechtgeschichte, das erst in jüngster Zeit als eigenständiges Forschungsfeld an Kontur gewinnt.

Dass mediengeschichtliche Ansätze in der Menschenrechtsgeschichte aktuell größere Aufmerksamkeit finden, zeigt vor allem die Publikation erster Sammelbände. ${ }^{34}$ Drei Aspekte sind hierbei von besonderem Interesse: Erstens gilt es, den Einfluss medialer Strukturen und technologischer Innovationen herauszuarbeiten, die die Arbeit von Menschenrechtsakteuren - aber auch von autoritären Regimen und anderen Menschenrechtsverletzern - signifikant verändert haben, etwa durch die Einführung von Fotografie, Fernsehen oder des Internets. Zweitens stehen die Bildsprachen und medialen Darstellungsweisen im Mittelpunkt, die im Feld von Menschenrechten und Humanitarismus oft erstaunlich lange Kontinuitätslinien aufwiesen - beispielsweise in der Darstellung körperlicher Leiden oder der Verwendung von Kindern als Bildmotiven. Drittens geht es hiermit verbunden um die Rolle von Populärkultur als ein Mittel, mit dem Menschenrechtsthemen an breitere Bevölkerungsgruppen kommuniziert wurden, dabei aber auch in ihrem Bedeutungsgehalt transformiert wurden. ${ }^{35}$

Eine der neueren Arbeiten, in der Erkenntnisse der Mediengeschichte für die Geschichte der Menschenrechte fruchtbar gemacht werden, ist Lasse Heertens Untersuchung über den Biafra-Konflikt. ${ }^{36}$ Heertens Dissertation ist die erste umfassende geschichtswissenschaftliche Darstellung des nigerianischen Bürgerkriegs, der von 1967

\footnotetext{
34 Fehrenbach, Heide/Rodogno, Davide (Hrsg.): Humanitarian Photography. A History, Cambridge UP, Cambridge 2015; Paulmann, Johannes (Hrsg.): Humanitarianism and Media. 1900 to the Present, Berghahn, Oxford/New York 2018. Demnächst erscheint darüber hinaus Hofmann, Birgit/Stallmann, Martin (Hrsg.): Menschenrecht als Nachricht. Medien, Öffentlichkeit und Moral seit dem 19. Jahrhundert, Campus, Frankfurt a. M./New York 2020.

35 Siehe hierzu z.B. Möckel, Benjamin: The Material Culture of Human Rights. Consumer Products, Boycotts and the Transformation of Human Rights Activism in the 1970s and 1980s, in: International Journal for History, Culture and Modernity 6 (2018), H. 1, S. 76-104.

36 Heerten, Lasse: The Biafran War and Postcolonial Humanitarianism. Spectacles of Suffering, Cambridge UP, Cambridge 2018.
} 
bis 1970 zwischen Nigeria und dem nach Unabhängigkeit strebenden südöstlichen Landesteil geführt wurde und ab Sommer 1968 für einige Monate auch die westlichen Nachrichten dominierte. Auch wenn Heerten die Bedeutung und vor allem die Singularität des Fallbeispiels an einigen Stellen zu stark betont und dadurch die Chance verpasst, das nigerianische Beispiel zu anderen zeitgenössischen Konflikten in Verbindung zu setzen, ist sein Buch ein empirisch umfassender und argumentativ überzeugender Beitrag zu neueren Forschungen der Menschenrechtsgeschichte und des Humanitarismus. Es wird in Zukunft zweifellos das Standardwerk zum BiafraKonflikt darstellen. Darüber hinaus ist sein Buch ein einleuchtendes Beispiel für neuere Ansätze, Menschenrechtsgeschichte nicht mehr als separiertes Forschungsfeld zu verstehen, sondern bewusst die Verbindungen zu verwandten Themenbereichen $\mathrm{zu}$ suchen und in eine umfassendere Gesellschaftsgeschichte internationaler Normen einzuordnen.

Heerten nähert sich seinem Thema multiperspektivisch, indem er anhand des zeitlich relativ eingegrenzten Konflikts (und dessen noch kürzerer Präsenz in den westlichen Medien) einerseits die Veränderungen postkolonialer Staatlichkeit sowie andererseits die Strukturmerkmale der westlichen Solidaritätsbewegungen herausarbeitet. Das erste Kapitel verdeutlicht zunächst die kolonialen Ursprünge des Konflikts, die insbesondere in den - sowohl räumlichen als auch ethnischen - Grenzziehungen der britischen Kolonialherrschaft zu suchen sind. Die beiden folgenden Kapitel beschreiben die Loslösung des Gebiets „Biafra“ von Nigeria sowie die Versuche der Separatisten, das Gebiet als eigenständigen Staat auf der internationalen Bühne zu etablieren: Zum einen auf völkerrechtlicher Ebene, indem man das Recht auf nationale Selbstbestimmung für sich reklamierte, und zum anderen auf medialer Ebene, indem man das Leid der eigenen Bevölkerung in den Vordergrund rückte und gegenüber Nigeria den Vorwurf eines Genozids erhob. Während Biafra mit dem Ziel der völkerrechtlichen Anerkennung auf ganzer Linie scheiterte, erwies sich das humanitäre Narrativ innerhalb der westlichen Öffentlichkeit als extrem erfolgreich - so sehr, dass es in letzter Konsequenz dem Streben der lokalen Akteure nach internationaler Anerkennung sogar entgegenlief.

Die hieraus entstehenden internationalen Solidaritätskampagnen stehen im Zentrum des Buches und werden von Heerten auf beeindruckender Quellengrundlage und in transnationaler Perspektive für Deutschland, Großbritannien, Frankreich und die USA analysiert. Nach einer Darstellung der ersten Berichte aus dem Sommer 1968 fokussiert er zunächst auf zwei Topoi, die hier besonders prominent wurden: erstens die sogenannten „Biafra-Babys“, die die Bildsprache des Konfliktes in zentraler Weise prägten, sowie zweitens die Vergleiche mit dem Holocaust, der immer wieder als semantische und ikonografische Referenz aufgegriffen wurde. Die letzten drei Kapitel beschäftigten sich mit dem Ende und den Nachwirkungen des Konfliktes, sowohl in militärischer Hinsicht als auch in Bezug auf das ebenso schnelle Verschwinden des Konfliktes aus dem Fokus der westlichen Öffentlichkeit.

Abgesehen von der inhaltlichen Darstellung ist Heertens Buch auch in konzeptioneller Hinsicht ein wichtiger Beitrag für die Menschenrechtsgeschichte, weil er bewusst darauf abzielt, die Grenzziehungen zwischen Menschenrechten, Humanitarismus und Entwicklungspolitik zu hinterfragen. Während viele Forschungen weiterhin klar zwischen diesen Feldern trennen, zeigt sich an Heertens Fallbeispiel sehr 
deutlich, dass die historischen Akteure selbst diesen Grenzziehungen kaum Bedeutung beimaßen und je nach Kontext und Opportunität humanitäre, entwicklungspolitische oder eben menschenrechtspolitische Argumente für die eigenen politischen Ziele nutzten. Die historische Forschung kann diese Diskurse nur adäquat erfassen, wenn sie auch selbst stärker nach den Schnittpunkten zwischen diesen Forschungsfeldern sucht. Hierfür ist Heertens Buch ein überzeugendes Beispiel.

Dabei ist Heertens mediengeschichtlicher Zugang besonders dort spannend, wo es ihm gelingt, sowohl die Analogien als auch die Differenzen herauszuarbeiten, die zwischen den humanitären Motiven und den menschenrechtspolitischen und völkerrechtlichen Argumenten bestanden. Wie Heerten zeigt, lag der Fokus der westlichen Medien eindeutig auf dem humanitären Narrativ, das eng an Darstellungen des (oft nackten, meist kindlichen, seltener weiblichen, fast nie männlichen) Körpers anschloss. Körperliches Leid stand im Zentrum dieser Bilder und hiervon abweichende Motive fanden kaum Aufnahme in die westliche Öffentlichkeit. Weitere Forschungen könnten hier vor allem die langen Traditionslinien dieser Darstellungsweisen herausarbeiten, die meist auf kolonialen Deutungsmustern beruhten. Naheliegende Anknüpfungspunkte wie die Darstellungen der Kongo-Gräuel wären zu nennen, aber auch weiter zurückliegende Muster der Effeminisierung und Infantilisierung kolonialer Räume und Bevölkerungen. Die Menschenrechtsgeschichte jedenfalls wird gut daran tun, solche Sichtweisen zu integrieren und eingespielte Deutungsmuster der ,savages, victims and saviors“ $\mathrm{zu}$ historisieren. ${ }^{37}$

Eine zweite Neuerscheinung zeigt, dass ein mediengeschichtlicher Ansatz auch geeignet ist, um eigentlich bekannten Themenfeldern neue Perspektiven abzugewinnen. Denn Mark Philip Bradleys Buch ,The World Reimagined“ 38 führt auf den ersten Blick in relativ bekannte Gefilde: Im Zentrum steht die US-amerikanische Menschenrechtsbewegung der Nachkriegszeit, die in zwei Hauptteilen für die 1940er und die 1970er Jahre untersucht wird. Dass hieraus dennoch kein altbekanntes Narrativ entsteht, liegt daran, dass Bradley einen eigenständigen Zugang wählt, mit dem er ,alten“ Forschungsdiskussionen an vielen Stellen neue Impulse verleihen kann. Vor allen Dingen gelingt es ihm, seinen zeitlichen und räumlichen Zugang jeweils auf konzeptionell anspruchsvolle Weise zu brechen. Zeitlich ist das Buch zwar anhand der etablierten Doppelrevolution der 1940er und 1970er Jahre strukturiert. Jedoch legt er seinen Schwerpunkt nicht auf die beiden Jahrzehnte als die in der Forschung etablierten ,kick-off moments“, sondern auf die zeitlich etwas vorgelagerten ,contrapunctual moments“ (S. 5), die für das historische Verständnis von ebenso großer Bedeutung seien. In räumlicher Perspektive wird der Blick auf die USA globalgeschichtlich gebrochen, indem Bradley die USA nicht als Avantgarde der Menschenrechtsbewegung, sondern im Gegenteil eher als einen Nachzügler interpretiert, dessen Debatten vor allem durch den Import global kursierender Ideen geprägt wurden.

\footnotetext{
37 Mutua, Makau: Savages, Victims, and Saviors. The Metaphor of Human Rights, in: Harvard International Law Journal 42 (2001), H. 1, S. 201-245.

38 Bradley, Mark Philip: The World Reimagined. Americans and Human Rights in the Twentieth Century, Cambridge UP, Cambridge 2016.
} 
Noch wichtiger sind die thematischen Schwerpunkte, die Bradley setzt. Sein Fokus liegt explizit nicht auf den Ebenen von Diplomatie, Recht und internationaler Politik. Stattdessen geht es ihm um die Frage, wie Menschenrechte zu einem ,everyday vernacular" (S. 3) wurden, also einer moralischen Leitvokabel, die von breiten Teilen der Bevölkerung geteilt und als sinnvoll aufgefasst wurde. Damit greift er ein grundlegendes Problem der Menschenrechtsgeschichte auf. Während zahlreiche Untersuchungen dargestellt haben, wie Menschenrechte in der zweiten Hälfte des 20. Jahrhundert zur lingua franca der internationalen Politik geworden sind, steht die Beantwortung der Frage, wie diese politischen und juristischen Diskurse ihren Weg in die Gesamtgesellschaft fanden, noch relativ am Anfang.

Sollen Menschenrechte in dieser Weise in ihrer gesamtgesellschaftlichen Bedeutung analysiert werden, ist es notwendig, den Blick auf jene Gesellschaftsbereiche auszuweiten, die nicht explizit zum Feld der Menschenrechtspolitik gehörten. Allerdings birgt dies zugleich die Gefahr, die heutige Ubiquität des Menschenrechtsdiskurses in die Vergangenheit zu projizieren und alle Felder, in denen sich Gesellschaften über Rechte, Pflichten und Normen austauschen, als Teil der Menschenrechtsgeschichte zu interpretieren. Bradley gelingt dieser Spagat über weite Strecken überzeugend. Einerseits erweitert er die Gruppe der Akteure, die er betrachtet: Neben Menschenrechtsaktivist_innen, Politiker_innen und Jurist_innen kommt bei ihm eine Gruppe von Menschen in den Blick, die er als ,human rights amateurs“ bezeichnet und zu denen er unter anderem Künstler_innen, Student_innen oder Journalist_innen zählt, die sich in unterschiedlicher Weise mit dem Themenfeld auseinandersetzten. Zweitens ist auch sein Quellenzugang weiter gefasst und beinhaltet ein breites Spektrum an Medien wie Fotografien, Filme, Romane, Ausstellungen, Reportagen, Musik und Straßentheater.

Im ersten Teil des Buches zu den 1940er Jahren entsteht aus diesem Ansatz eine äußerst aufschlussreiche und originelle Perspektiverweiterung. Bradley konzentriert sich auf drei Kontexte, die jeweils neue Sichtweisen auf den Menschenrechtsdiskurs der amerikanischen Nachkriegszeit eröffnen. Das erste Kapitel interpretiert die sozialdokumentarische Fotografie der New Deal-Periode als einen Ausgangspunkt, an dem nach Bradley eine neue „,sensibility“ (S. 14) entstanden sei, an die der Menschenrechtsdiskurs der 1940er Jahre habe anschließen können. Hier überträgt der Autor Lynn Hunts Thesen zur Genese der Menschenrechte im 18. Jahrhundert in produktiver Weise in das 20. Jahrhundert, und zeigt, dass auch Bilder wie Dorothea Langes „Migrant Mother“ als Quellen der Menschenrechtsgeschichte verstanden werden können. Das zweite Kapitel beschäftigt sich mit den Debatten über ökonomische Rechte in den USA der Kriegsjahre, wobei Bradley zu einer sehr viel positiveren Einschätzung der Reichweite dieser Diskurse gelangt als Samuel Moyn in „Not Enough“. Den dritten Kontext bilden die atrocity pictures der Nachkriegszeit, vor allem aus den befreiten Konzentrationslagern. Dieses Kapitel verdeutlicht, dass die oft betonte These, die Genese der Menschenrechte in den 1940er Jahren sei kaum mit den Ereignissen des Holocaust verbunden gewesen, in mediengeschichtlicher Perspektive zu überdenken ist. Bradley zeigt überzeugend, dass die globale Zirkulation dieser Bilder gerade im Moment der Gründungskonferenz der UN in San Francisco einen wichtigen Zusammenhang darstellten. Er macht aber auch deutlich, dass die Bilder zu diesem Zeitpunkt noch nicht Teil einer - damals noch 
gar nicht existierenden - Holocausterinnerung waren, sondern in den USA in eigene Bild- und Deutungstraditionen eingefügt wurden - einerseits in die weit verbreiteten Bilder von Lynchmorden in den USA in den Jahrzehnten zuvor, andererseits in die Diskussionen über die Internierung japanischstämmiger Amerikaner_innen während des Krieges.

Eröffnet Bradleys Ansatz für die 1940er Jahre viele neue Perspektiven, so folgt der zweite Teil zu den 1970er Jahren etablierten Interpretationsmustern. Sein mediengeschichtlicher Ansatz besitzt hier ein sehr viel geringeres Überraschungsmoment, und auch die globalgeschichtliche Einbettung ist im Ganzen weniger überzeugend als im ersten Teil. Während Bradley für die 1940er Jahre sehr erhellend die Partikularitäten und lokalen Kontexte des amerikanischen Fallbeispiels aufzeigen kann, tendiert der zweite Teil eher zu einer Rezeptionsgeschichte globaler Entwicklungen innerhalb der USA.

Seine zentrale These ist, dass die Vereinigten Staaten in den 1970er Jahren als Nachzügler der Menschenrechtsrevolution zu verstehen sind, der mehr als Rezipient denn als eigenständiger Akteur agierte. Hierfür zeichnet Bradley zunächst zentrale Entwicklungen der 1960er und 1970er Jahre nach, in denen Menschenrechte in einer globalen Zirkulation von Ideen zu einer neuen Leitlinie der internationalen Politik wurden. Anschließend greifen die beiden folgenden Kapitel zwei Kontexte auf, in denen diese Diskurse besonders intensiv in den amerikanischen Diskussionen aufgenommen wurden - und sich dabei immer auch mit Fragen der nationalen Politik und von gesamtgesellschaftlichen Selbstverständigungsprozessen verbanden. Das gilt einerseits für die Beschäftigung mit den osteuropäischen Dissidenten, insbesondere mit dem seit 1976 in den USA im Exil lebenden Alexander Solschenizyn, sowie zweitens für die Auseinandersetzung mit den autoritären Diktaturen in Lateinamerika. Im Mittelpunkt des letzten Kapitels steht die Frage, welche spezifischen Formen des Menschenrechtsdiskurses in diesem Kontext in den USA entstanden, wobei Bradley unter anderem auf die Unterschiede zwischen den europäischen und der amerikanischen Sektion von Amnesty International als Beispiel verweist.

Insgesamt ist auch der zweite Teil des Buches eine gut geschriebene und klar argumentierte Darstellung der gesellschaftlichen Bedeutung der Menschenrechte für die amerikanische Politik und Gesellschaft. Dass dieser Teil weniger Überraschungsmomente beinhaltet, liegt wohl in erster Linie daran, dass die von Bradley fokussierten gesamtgesellschaftlichen Einbettungen des Menschenrechtsdiskurses für diesen Zeitraum schon stärker in die Forschung integriert sind, als dies bislang für die 1940er Jahre der Fall war. Dennoch ist gerade die von Bradley forcierte Ausweitung der Quellen- und Themenbasis auf Felder, in denen Menschenrechte nur implizit eine Rolle spielen, ein wichtiger Anknüpfungspunkt für zukünftige Forschungen.

\section{Menschenrechte und die Herausforderungen der Globalgeschichte}

Ein viertes Feld, das die Menschenrechtsgeschichte zuletzt stark geprägt hat und in den nächsten Jahren weitere wichtige Forschungen hervorbringen wird, ist die Globalgeschichte. Globalgeschichtliche Ansätze sind für die Menschenrechtsgeschichte besonders aus zwei Gründen von Bedeutung: Einerseits gilt es, die Interpretation 
der Menschenrechte als eine im Kern westliche Vorstellung infrage zu stellen und den Einfluss nichtwestlicher Akteure sowie insbesondere die globale Zirkulation von Ideen und Konzepten aufzuzeigen. Zugleich ist eine globalgeschichtliche Perspektive aber auch geeignet, um die immer wieder proklamierte Universalität der Menschenrechte kritisch zu reflektieren und auf ihre realen Machtasymmetrien und Deutungshierarchien zu hinterfragen. ${ }^{39}$

In den bislang erschienenen Arbeiten zur globalen Dimension der Menschenrechte haben vor allem zwei Fragekomplexe im Vordergrund gestanden: zum einen die Verbindung zwischen Menschenrechten, Kolonialismus und Dekolonisation. Hier hat insbesondere Roland Burke eine wegweisende Arbeit veröffentlicht. ${ }^{40}$ Ein zweiter, damit verbundener Aspekt hat sich mit der Frage nach dem ,Selbstbestimmungsrecht der Völker“ als Menschenrecht auseinandergesetzt. Dazu hat Jörg Fisch eine wichtige Gesamtdarstellung vorgelegt. ${ }^{41}$ Mittlerweile lässt sich jedoch beobachten, dass globalgeschichtliche Ansätze sehr viel selbstverständlicher in neuere Forschungsarbeiten integriert werden. Zuletzt haben beispielsweise mehrere Studien die wichtige Bedeutung jüdischer Aktivist_innen und Jurist_innen herausgearbeitet, die in vielen Fällen Nationengrenzen überschritten und in besonderem Maße die Bedeutung internationaler Netzwerke und Austauschprozesse für die Menschenrechtsbewegung im 20. Jahrhundert verdeutlichen. ${ }^{42} \mathrm{Im}$ Zusammenhang mit einer Reorientierung der Menschenrechtsgeschichte und der Kritik an einem allein auf den Westen fokussierten Blick auf die Menschenrechte sind außerdem auch die osteuropäischen Erfahrungen und Kontexte wieder stärker ins Blickfeld gerückt. Neben den oben zitierten Publikationen zur DDR gilt das zum Beispiel für die Forschungen von Celia Donert, Christie Miedema und Benjamin Nathans, der aktuell an einer Gesamtdarstellung der sowjetischen Dissidentenbewegung arbeitet. ${ }^{43}$ Im Folgenden werden zwei Arbeiten genauer vorgestellt, die auf unterschiedliche Weise auch konzeptionell an die Forschungsdiskussionen der Globalgeschichte anschließen und explizit eine postkoloniale Dimension ins Zentrum der eigenen Argumentation stellen.

Die erste Veröffentlichung stammt von Steven Jensen, der derzeit am Danish Institute for Human Rights arbeitet. ${ }^{44}$ Sein Ansatz ist dezidiert revisionistisch. Die

39 Siehe explizit zu dieser Frage Joas, Hans: Sind die Menschenrechte westlich?, Kösel, München 2015.

40 Burke: Decolonization (wie Anm. 18). Siehe auch Klose: Menschenrechte (wie Anm. 18).

41 Fisch, Jörg: Das Selbstbestimmungsrecht der Völker. Die Domestizierung einer Illusion, Beck, München 2010.

42 Loeffler, James: Rooted Cosmopolitans. Jews and Human Rights in the Twentieth Century, Yale UP, New Haven, CT/London 2018; Paz, Reut Yael: A Gateway Between a Distant God and a Cruel World. The Contribution of Jewish German-Speaking Scholars to International Law, Nijhoff, Dordrecht u. a. 2013; Loeffler, James/Paz, Moria (Hrsg.): The Law of Strangers. Jewish Lawyers and International Law in the Twentieth Century, Cambridge UP, Cambridge 2019.

43 Donert, Celia: The Rights of the Roma. The Struggle for Citizenship in Postwar Czechoslovakia, Cambridge UP, Cambridge 2017; Miedema, Christie: Not a Movement of Dissidents. Amnesty International Beyond the Iron Curtain (Schriftenreihe Menschenrechte im 20. Jahrhundert, Bd. 4), Wallstein, Göttingen 2019; Nathans, Benjamin: Talking Fish. On Soviet Dissident Memoirs, in: The Journal of Modern History 87 (2015), H. 3, S. 579-614.

44 Jensen, Steven L. B.: The Making of International Human Rights. The 1960s, Decolonization, and the Reconstruction of Global Values, Cambridge UP, Cambridge 2016. 
Geschichte der Menschenrechte, so sein an mehreren Stellen des Buches wiederholtes Argument, sei bislang fast ausschließlich aus der Perspektive westlicher Staaten und Akteure erzählt und der Einfluss von Akteuren des Globalen Südens auf diese Weise systematisch marginalisiert worden. Dies werde der eigentlichen Entwicklungsdynamik jedoch nicht gerecht. In Wirklichkeit, so Jensen, seien es vor allem Akteure aus dem Globalen Süden gewesen, die als „lead proponents“ (S. 2) die Menschenrechte auf dem Feld der internationalen Politik etabliert hätten.

Diese Argumente trägt der Autor mit dem Anspruch einer völligen Neuinterpretation der existierenden Forschung vor. Das schießt an mehreren Stellen über das Ziel hinaus, was jedoch verständlich ist, wenn man wie Jensen den Anspruch erhebt, ein Forschungsfeld neu besetzen $\mathrm{zu}$ wollen. Und Jensen hat genug intellektuelle Kraft und argumentative Schärfe, um sein Buch in der Tat als eine Intervention in das Forschungsfeld zu positionieren, an der man in Zukunft nicht vorbeikommen wird. Allerdings ist sein mehrfach wiederholter Anspruch, hiermit ein völlig neues Feld der Menschenrechtsgeschichte zu erschließen, zu relativieren. Im Gegenteil erscheint es an vielen Stellen als eine zentrale Schwäche des Buches, dass sich der Autor an keiner Stelle substanziell mit den durchaus existierenden Forschungen zur Globalgeschichte der Menschenrechte - etwa von Roland Burke, Fabian Klose oder Simon Stevens - auseinandersetzt. Gerade mit der Forderung, globalgeschichtlichen Ansätzen in der Menschenrechtsgeschichte eine größere Bedeutung zukommen zu lassen, wäre es wünschenswert gewesen, diese Ansätze stärker aufzugreifen und kritisch fortzuschreiben.

Betrachtet man den empirischen Teil des Buches genauer, dann steht vor allem Folgendes im Mittelpunkt: Jensen möchte in seiner Arbeit den Einfluss und die Aktivitäten von kleineren Staaten insbesondere aus dem Globalen Süden stärker in den Blick nehmen und herausarbeiten, welche Rolle diese Staaten in der Formulierung neuer Themenfelder und Konventionen gespielt haben. Im Zentrum steht die Menschenrechtsarbeit innerhalb der UN - ein Bereich also, der in der Forschung schon intensiv betrachtet worden ist. Neu ist jedoch, wie Jensen überzeugend aufzeigt, dass die Initiativen der Staaten des Globalen Südens nicht allein instrumentell im Kontext des antikolonialen Befreiungskampfes zu verstehen sind, sondern die Menschenrechtsarbeit der UN mit einer klaren und eigenständigen Agenda voranbrachten. Im Zentrum steht dabei das Beispiel Jamaika, das nach seiner Unabhängigkeit und unter der Ägide ihres äußerst engagierten UN-Botschafters Egerton Richardson die Diskussionen über die Rolle der Menschenrechte in der UN in großem Maße mitprägte und - so Jensens These - auf diese Weise überhaupt am Leben hielt. Das Engagement Jamaikas und einiger anderer Staaten interpretiert er denn auch als Brücke zwischen den frühen Menschenrechtsinitiativen der Nachkriegszeit und deren Renaissance in den 1970er Jahren.

Das Kapitel zu Jamaika bildet die empirische Basis für Jensens Neujustierung des Einflusses unterschiedlicher Akteure und Staaten innerhalb der internationalen Menschenrechtspolitik. In den beiden folgenden Kapiteln wird dies anhand von zwei UN-Konventionen weiter konkretisiert: einerseits an der 1965 verabschiedeten „Convention on the Elimination of all Forms of Racial Discrimination“, die Jensen als eine entscheidende Wegmarke zu den rechtlich bindenden Menschenrechtspakten im darauffolgenden Jahr interpretiert, sowie andererseits an der letztlich nicht 
verabschiedeten „Convention on the Elimination of all Forms of Religious Intolerance“, über die zur selben Zeit in der UN verhandelt wurde. Diese drei Kapitel sind die empirisch überzeugendsten, weil sie die konzeptionellen Thesen des Buches mit einer detaillierten, in erster Linie rechtsgeschichtlichen Diskussion der genannten UN-Initiativen verbinden. Jensen schafft es hier, ein spannendes Panorama der UNDiskussionen der 1960er Jahre zu entwerfen und auf diese Weise die Bedeutung dieser Dekade für die Menschenrechtsgeschichte zu stärken. Zwar ist es vermutlich übertrieben, den Zeitraum als eine ,vergessene Dekade“ (S. 2) der Menschenrechtsgeschichte zu bezeichnen, da sowohl die beiden genannten Konventionen als auch die Menschenrechtspakte von 1966 in der Forschung große Aufmerksamkeit gefunden haben. Dennoch gelingt es ihm, den Fokus auf die 1970er Jahre zum Teil zu hinterfragen und auf die wichtigen, vor allem juristischen, Entwicklungsschritte in den Jahren davor zu verweisen.

Demgegenüber ist die Argumentation der folgenden Kapitel weniger klar. Für Jensens Argument, dass in Wirklichkeit die 1960er Jahre als Durchbruch der Menschenrechte $\mathrm{zu}$ verstehen sind, ist es von zentraler Bedeutung, zu zeigen, dass die dort initiierten Debatten auch den Menschenrechtsdiskurs der 1970er Jahre mitbestimmten. Dies gelingt ihm jedoch nur sehr eingeschränkt. Ein Kapitel beschäftigt sich mit der ersten UN-Menschenrechtskonferenz in Teheran im Jahr 1968. Hier kann er zwar einige neue Akzente setzen, letztlich geben seine Ausführungen aber kaum Anlass, die in der Forschung etablierte Interpretation der Konferenz als Rückschritt und Misserfolg substanziell zu revidieren. Das daran anschließende Kapitel behandelt den KSZE-Prozess und die Helsinki-Schlussakte. Jensen versucht hier zu zeigen, wie stark diese Verhandlungen an Debatten und Initiativen des Globalen Südens innerhalb der UN anschlossen. Auch dies ist im Detail interessant. Seine These aber, dass die KSZE-Initiativen in erster Linie auf diesen Debatten beruhten, ist wenig überzeugend. Der letzte Teil spannt dann unter dem Titel ,The Presence of the Disapeared“ einen langen Bogen bis in die 1990er Jahre, der zwar als Einzelkapitel anregend ist, aber letztlich relativ unverbunden zu den vorherigen Ausführungen bleibt.

In den konkreten Darstellungen enthält Jensens Arbeit somit viele neue Einsichten, die zukünftige Forschungen inspirieren werden. Weniger überzeugend ist das Buch überall dort, wo der Autor diese Erkenntnisse zu nutzen versucht, um ein eigenständiges und in sich geschlossenes Gegennarrativ zu einer vermeintlich allein in westlichen und europäischen Narrativen gefangenen Meistererzählung der Menschenrechtsgeschichte zu entwerfen. Er konstruiert dabei eine sehr dichotomische Gegenüberstellung, die der existierenden Forschungsdiskussion nicht gerecht wird. Dies ist insofern legitim, als sein Buch auch als Intervention in aktuelle Forschungsdiskussionen $\mathrm{zu}$ verstehen ist und dafür auch mit strategisch eingesetzten Zuspitzungen operiert. Wichtiger ist, dass dies auch konzeptionell einem globalgeschichtlichen Ansatz nur zum Teil entspricht. So besteht die zentrale Herausforderung eines globalgeschichtlichen Blicks gerade darin, neue Narrative zu entwickeln, die ohne ein fest definiertes Zentrum auskommen. Statt neue Staaten als ,lead proponents“ einzuführen beziehungsweise für weitere Jahrzehnte den „breakthrough“ der Menschenrechte zu postulieren (S. 2, 4, 5), scheint die eigentliche Aufgabe für künftige Forschungen gerade darin zu liegen, die Multiplizität von Orten, Zeiten, 
Akteuren und Themen in den Blick zu nehmen und auf diese Weise unseren Sinn für Gleichzeitigkeiten, Deutungskonkurrenzen und Widersprüche zu schärfen - eine sowohl analytische als auch narrative Herausforderung.

Überzeugender ist in dieser Hinsicht die Dissertation von Simon Stevens, der sich in globalgeschichtlicher Perspektive mit den Kampagnen gegen das südafrikanische Apartheidregime auseinandersetzt. ${ }^{45}$ Stevens nimmt damit ein Thema in den Blick, das in den vergangenen Jahren schon intensiv - und auch transnational vergleichend - untersucht worden ist. ${ }^{46}$ Allerdings konzentriert er sich vor allem auf die ersten drei Nachkriegsjahrzehnte, also jenen Zeitraum, bevor die westlichen AntiApartheid-Bewegungen den Zenit ihrer Aktivitäten erreichten und damit auch die Deutungshoheit über die meisten Kampagnen erlangten. Im Gegensatz hierzu zeigt Stevens, wie weitgefasst und heterogen die Initiativen gegen das südafrikanische Regime in der Anfangsphase waren und wie wichtig die Rolle afrikanischer und anderer nichtwestlicher Akteure in diesem Kontext war.

Der zeitliche Rahmen ist mit der zentralen Ausgangsfrage der Arbeit verbunden. Während die meisten Studien zur Anti-Apartheid-Bewegung die Boykottkampagnen als wichtigste Proteststrategie als gegeben voraussetzen, analysiert Stevens, wann und warum sich die Bewegung in so starkem Maße auf Formen des Konsumboykotts und der Forderung nach Sanktionen konzentrierte. Einerseits zeigt er dabei, dass die frühen Kampagnen noch keineswegs auf diese beiden Protestformen festgelegt waren und ein sehr viel breiteres Spektrum an Proteststrategien verfolgten. Andererseits verdeutlicht er, in welch starkem Maße die Boykottkampagnen aus dem südafrikanischen Kontext selbst hervorgingen sowie längere Traditionen antikolonialer Boykotte im Globalen Süden aufgriffen, etwa der Swadeshi-Bewegung in Indien oder antikolonialer Proteste in anderen Ländern Afrikas.

Die Arbeit ist in vier chronologische Kapitel strukturiert. Das erste Kapitel stellt die 1940er und 1950er Jahre in den Mittelpunkt und analysiert die ersten internationalen Kampagnen gegen Südafrika. Diese gingen nicht von westlichen Staaten aus, sondern wurden von Indien initiiert, das damit die Behandlung der indischen Minderheit in Südafrika zum Thema machte. Indien verhängte als erstes Land überhaupt Sanktionen gegen Südafrika und es gelang dem Land auch, die Situation der indischen Minderheit auf die Agenda der ersten Generalversammlung der UN zu setzen, was die lange Geschichte der Beschäftigung mit Südafrika innerhalb der UN begründete. In einem weiteren Abschnitt stellt Stevens die Proteste der lokalen Bevölkerung Südafrikas dar und verdeutlicht, wie sich das Instrument des Boykotts schon in den 1940er Jahren - etwa im Kampf für bessere Arbeitsbedingungen oder

\footnotetext{
45 Die Dissertation von Simon Stevens trägt den Titel „Boycotts and Sanctions Against South Africa. An International History, 1946-1970“ und wurde 2016 an der Columbia University (New York) eingereicht. Sie ist über folgende Seite online zugänglich: URL: <https://academiccommons.columbia.edu/doi/ 10.7916/D8HT2P74> [Zugriff: 20.08.2019]. Eine überarbeitete Buchveröffentlichung ist angekündigt und trägt den Arbeitstitel: Stevens, Simon: Laying Siege to South Africa. Anti-Apartheid Boycotts and Sanctions, and the Transformation of Global Politics.

46 Siehe z. B. Konieczna, Anna/Skinner, Rob (Hrsg.): A Global History of Anti-Apartheid. 'Forward to Freedom' in South Africa, Palgrave Macmillan, Basingstoke/New York 2019; sowie demnächst Andresen, Knud/Justke, Sebastian/Siegfried, Detlef (Hrsg.): Apartheid in Western Europe. Perceptions and Reactions, 1948-1994, Palgrave Macmillan, Basingstoke/New York [voraussichtlich 2020].
} 
gegen Preiserhöhungen im öffentlichen Nahverkehr - als Protestmittel etablierte. Vor allem hierauf rekurrierten die späteren Boykottaufrufe des African National Congress (ANC) und anderer südafrikanischer Akteure.

Das zweite Kapitel analysiert auf dieser Basis im Detail die Boykottaufrufe von ANC und Pan Africanist Congress (PAC) im Jahr 1959, die als Ausgangspunkt der internationalen Boykottbewegung gelten. Besonders hier stellt Stevens' globaler und transnational vergleichender Blick eine spannende Neuinterpretation der Genese der internationalen Boykottbewegung dar. Stevens deutet den meist als Ursprungspunkt angeführten direkten Boykottaufruf des ANC an seine britischen Partner als „Gründungsmythos" (S. 134) und relativiert im Ganzen die Bedeutung des ANC für die Entstehung der Boykottkampagne. Bedeutsamer war in seiner Interpretation der erste All African Congress in Accra im Jahr 1958, auf dem vor allem auf Initiative von Michael Scott Sanktionen aller afrikanischer Staaten gegenüber Südafrika beschlossen wurden. Gleichzeitig habe auch der ANC in Südafrika mit Boykottkampagnen experimentiert, die jedoch zunächst allein als nationale Protestaktionen gedacht waren. Erst nach 1960, als in der Folge des Sharpeville-Massakers der ANC verboten wurde und viele Aktivist_innen ins Exil gingen, bekamen die internationalen Solidaritätsbewegungen größere Bedeutung. Auf dieser Grundlage entstand zwischen 1960 und 1964 das Fundament der internationalen Anti-Apartheid-Bewegung. Dies ist das Thema des dritten Hauptkapitels. Das abschließende vierte Kapitel betrachtet die Jahre zwischen 1964 und 1970. Hier steht die Frage im Mittelpunkt, warum sich diese Bewegungen relativ schnell auf das Mittel des Konsumboykotts konzentrierten. Nach Stevens hätten in den ersten Jahren zunächst politische Kampagnen im Fokus gestanden, die einerseits auf die eigenen Regierungen, andererseits auf die UN abgezielt hätten. Erst als sich diese Kampagnen als wenig erfolgreich erwiesen, hätten sich die Protestbewegungen auf die Unternehmen als Kampagnenziel konzentriert und die Strategie des individuellen Konsumboykotts ins Zentrum gestellt.

Stevens gelingt es mit seiner Arbeit überzeugend, die meist als gegeben angenommene Wahl des Konsumboykotts als Protestmittel zu historisieren und die komplexen transnationalen Austauschprozesse aufzuzeigen, die zur Entstehung der Anti-Apartheid-Bewegung führten. Zugleich ist seine Arbeit auch auf theoretischmethodischer Ebene überzeugend. Im Kern erfüllt er die Forderung der Globalgeschichte, Akteuren, Dingen und Ideen zu folgen, ohne räumliche oder institutionelle Kontexte als gegebene Untersuchungsfelder vorauszusetzen. Daher spielen bei ihm auch westliche Akteure eine wichtige Rolle. Deren Analyse führt aber eben nicht zu einer teleologischen Erzählung der westlichen Protest- und Boykottbewegungen. Stattdessen verdeutlicht seine Arbeit die Heterogenität der Orte und Akteure, die in den 1940er bis 1960er Jahren die Kampagnen gegen Südafrika prägten. Dadurch kann seine Studie dazu beitragen, diese Heterogenität auch für die Zeit ab den 1970er Jahren wiederzuentdecken. So hat zum Beispiel Elizabeth Williams jüngst für den britischen Fall gezeigt, wie wichtig die Rolle vor allem der südafrikanischen Exilantinnen und anderer Einwanderergruppen in den frühen Kampagnen der AntiApartheid-Bewegung war, und wie diese Akteure dann in den folgenden Jahrzehnten 
innerhalb der Protestbewegungen weitgehend marginalisiert wurden. ${ }^{47}$ Eine solche Infragestellung etablierter Deutungen durch eine globalgeschichtliche Neuperspektivierung bildet das größte Erkenntnispotenzial neuer globalgeschichtlicher Arbeiten, die für die kommenden Jahre zu erwarten sind.

\section{Fazit und Forschungsperspektiven}

Was immer man von den aktuellen Krisendiagnosen halten mag, eines scheint sicher: Das Ende der Menschenrechte wird nicht das Ende der Menschenrechtsgeschichte bedeuten. Im Gegenteil: Alles deutet darauf hin, dass das Feld auch in den kommenden Jahren ein zentraler Bereich der Geschichtswissenschaft bleibt. Abschließend lassen sich provisorisch drei Forschungsperspektiven benennen, die für die folgenden Jahre relevant bleiben werden. Sie verweisen nicht zuletzt darauf, dass die drei Leitlinien, die oben als zentrale Merkmale der Menschenrechtsgeschichte in den 2000er und 2010er Jahren herausgearbeitet worden sind, sukzessive an Bedeutung $\mathrm{zu}$ verlieren scheinen.

Erstens lässt sich erkennen, dass Fragen der Periodisierung, die in den frühen Debatten eine so entscheidende Rolle gespielt haben, in letzter Zeit unwichtiger werden. Statt in einer an einzelnen Dekaden orientieren Suche nach den Ursprüngen, besteht die zentrale Herausforderung aktuell eher darin, die Pluritemporalitäten der Menschenrechtsgeschichte adäquat in den Blick zu nehmen. Begriffsgeschichtliche Ansätze werden hier zu anderen Periodisierungen kommen als praxeologische, institutionengeschichtliche zu anderen als mediengeschichtliche. Zugleich kommen mit der globalgeschichtlichen Perspektive noch einmal ganz neue Zeitlichkeiten in den Blick. Auch hier wird es vor allem darum gehen, anhand konkreter Akteure und Themenfelder Kontinuitäten und Diskontinuitäten herauszuarbeiten. Hierin liegen für kommende Forschungen und Gesamtdarstellungen sowohl analytische als auch narrative Herausforderungen.

Zweitens ist zu vermuten, dass auch das Streben nach einer möglichst klaren Abgrenzung der Menschenrechte von verwandten Konzepten und Forschungsfeldern an Bedeutung verlieren wird zugunsten eines Ansatzes, der die Überschneidungen und Austauschprozesse stärker betont. Besonders die Grenzen zwischen der Geschichte der Menschenrechte, des Humanitarismus und der Entwicklungspolitik, die in den ersten Jahren bewusst scharf gezogen worden sind, werden hierbei voraussichtlich aufgeweicht und zum Teil infrage gestellt werden. Samuel Moyns Buch ist hierfür ein Beispiel, und auch Lasse Heerten diskutiert diese Problematik explizit. Man kann annehmen, dass die spannendsten Arbeiten in nächster Zeit wohl genau an den Schnittstellen zwischen Menschenrechten, Humanitarismus und Entwicklungspolitik entstehen werden.

Drittens schließlich deutet sich seit längerer Zeit an, dass sich die neueren Forschungen noch mehr als bislang von dem Fokus auf politik- und diplomatiegeschichtliche sowie ideen- und begriffsgeschichtliche Zugänge lösen und vor allem

\footnotetext{
47 Williams, Elizabeth: The Politics of Race in Britain and South Africa. Black British Solidarity and the
} Anti-Apartheid Struggle, Tauris, London/New York 2015. 
medien- und kulturgeschichtliche, aber auch sozial- und wirtschaftsgeschichtliche Aspekte stärker integrieren. Die Geschichte der Menschenrechte wird sich hierbei vermutlich zunehmend von den konkreten Akteuren des eigenen Feldes lockern und andere Themenfelder auf ihren Einfluss auf die Menschenrechtsgeschichte befragen. Das gilt beispielsweise für die Konsumgeschichte, die Wissensgeschichte oder die Geschlechtergeschichte, die zuletzt größere Aufmerksamkeit gefunden hat. ${ }^{48} \mathrm{Im}$ Zentrum steht die Herausforderung, die Geschichte der Menschenrechte in eine Gesellschaftsgeschichte internationaler Normen einzufügen.

\section{Auswahlbibliografie}

- Bradley, Mark Philip: The World Reimagined. Americans and Human Rights in the Twentieth Century, 320 S., Cambridge UP, Cambridge 2016.

- Duranti, Marco: The Conservative Human Rights Revolution. European Identity, Transnational Politics, and the Origins of the European Convention, 528 S., Oxford UP, Oxford u. a. 2017.

- Frei, Norbert/Stahl, Daniel/Weinke, Annette (Hrsg): Human Rights and Humanitarian Intervention. Legitimizing the Use of Force Since the 1970s (Schriftenreihe Menschenrechte im 20. Jahrhundert, Bd. 2), 248 S., Wallstein, Göttingen 2017.

- Heerten, Lasse: The Biafran War and Postcolonial Humanitarianism. Spectacles of Suffering, 398 S., Cambridge UP, Cambridge 2018.

- Jensen, Steven L. B.: The Making of International Human Rights. The 1960s, Decolonization, and the Reconstruction of Global Values, 334 S., Cambridge UP, Cambridge 2016.

- Klose, Fabian: „In the Cause of Humanity“. Eine Geschichte der humanitären Intervention im langen 19. Jahrhundert, 516 S., Vandenhoeck \& Ruprecht, Göttingen 2019.

- Moyn, Samuel: Not Enough. Human Rights in an Unequal World, 296 S., Harvard UP, Cambridge, MA/London 2018.

- Stevens, Simon Murray: Boycotts and Sanctions Against South Africa: An International History, 1946-1970, 367 S. Zugl.: New York, Columbia University, Diss., 2016.

Funding Open Access funding provided by Projekt DEAL.

Open Access Dieser Artikel wird unter der Creative Commons Namensnennung 4.0 International Lizenz veröffentlicht, welche die Nutzung, Vervielfältigung, Bearbeitung, Verbreitung und Wiedergabe in jeglichem Medium und Format erlaubt, sofern Sie den/die ursprünglichen Autor(en) und die Quelle ordnungsgemäß nennen, einen Link zur Creative Commons Lizenz beifügen und angeben, ob Änderungen vorgenommen wurden.

\footnotetext{
48 Sachse, Carola: Leerstelle: Geschlecht. Zur Kritik der neueren zeithistorischen Menschenrechtsforschung, in: L'Homme. Europäische Zeitschrift Für Feministische Geschichtswissenschaft 25 (2014), H. 1, S. 103-212; Birke, Roman/Sachse, Carola (Hrsg.): Menschenrechte und Geschlecht im 20. Jahrhundert. Historische Studien (Diktaturen und ihre Überwindung im 20. und 21. Jahrhundert, Bd. 12), Wallstein, Göttingen 2018.
} 
Die in diesem Artikel enthaltenen Bilder und sonstiges Drittmaterial unterliegen ebenfalls der genannten Creative Commons Lizenz, sofern sich aus der Abbildungslegende nichts anderes ergibt. Sofern das betreffende Material nicht unter der genannten Creative Commons Lizenz steht und die betreffende Handlung nicht nach gesetzlichen Vorschriften erlaubt ist, ist für die oben aufgeführten Weiterverwendungen des Materials die Einwilligung des jeweiligen Rechteinhabers einzuholen.

Weitere Details zur Lizenz entnehmen Sie bitte der Lizenzinformation auf http://creativecommons.org/ licenses/by/4.0/deed.de.

Dr. Benjamin Möckel ist Akademischer Rat auf Zeit am Historischen Institut der Universität zu Köln. Von 2009 bis 2013 hat er in Göttingen und London seine Dissertation über die Kriegsjugendgenerationen des Zweiten Weltkriegs in den beiden deutschen Nachkriegsgesellschaften geschrieben. Seit 2014 arbeitet er - unterbrochen von einem einjährigen Aufenthalt in Oxford - an der Universität zu Köln an seiner Habilitation, die sich mit der Geschichte von Konsumprotesten und ethischer Konsumpraktiken in Großbritannien und der Bundesrepublik seit den 1960er Jahren beschäftigt. 